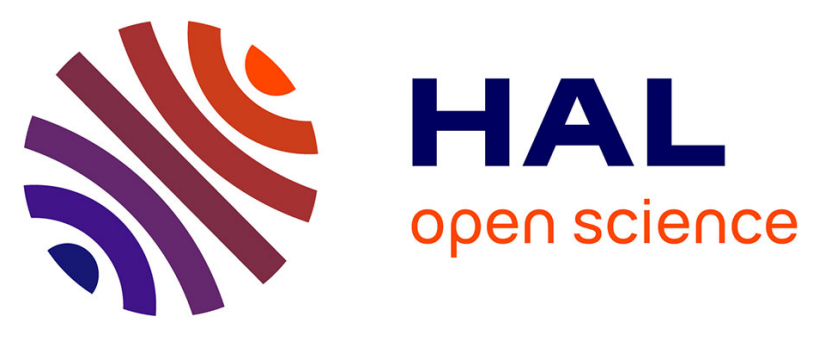

\title{
In Vitro Tyrosinase Inhibitory and Antioxidant Activities of Extracts and Constituents of Paeonia lactiflora Pall. Flowers
}

Abdulmagid Alabdul Magid, Marie Schmitt, Pierre-Charles Prin, Laure Pasquier, Laurence Voutquenne-Nazabadioko

\section{To cite this version:}

Abdulmagid Alabdul Magid, Marie Schmitt, Pierre-Charles Prin, Laure Pasquier, Laurence Voutquenne-Nazabadioko. In Vitro Tyrosinase Inhibitory and Antioxidant Activities of Extracts and Constituents of Paeonia lactiflora Pall. Flowers. The Natural Products Journal, 2017, 7 (3), 10.2174/2210315507666170509130921 . hal-01834118

\section{HAL Id: hal-01834118 \\ https://hal.univ-reims.fr/hal-01834118}

Submitted on 25 Nov 2021

HAL is a multi-disciplinary open access archive for the deposit and dissemination of scientific research documents, whether they are published or not. The documents may come from teaching and research institutions in France or abroad, or from public or private research centers.
L'archive ouverte pluridisciplinaire HAL, est destinée au dépôt et à la diffusion de documents scientifiques de niveau recherche, publiés ou non, émanant des établissements d'enseignement et de recherche français ou étrangers, des laboratoires publics ou privés. 


\title{
In vitro tyrosinase inhibitory and antioxidant activities of extracts and constituents of Paeonia lactiflora Pall. flowers
}

\author{
Abdulmagid Alabdul Magid ${ }^{* a}$, Marie Schmitt ${ }^{\mathrm{a}}$, Pierre-Charles Prin ${ }^{\mathrm{a}}$, Laure Pasquier ${ }^{\mathrm{b}}$, Laurence \\ Voutquenne-Nazabadioko ${ }^{a}$
} ${ }^{a}$ ICMR-UMR CNRS 7312, Groupe Isolement et Structure, Bât. 18, BP 1039, 51687 Reims Cedex 2, France, ${ }^{b}$ LVMH recherche,
département Innovation Ethnobotanique, 185 avenue de Verdun, 45800 Saint-Jean-de-Braye, France

\begin{abstract}
The aim of this study was to investigate the tyrosinase inhibitory and antioxidant activities of the extracts and constituents of Paeonia lactiflora Pall flowers 'Madame de Verneville'. The 70\% ethanol extract was purified by combination of chromatographic methods to afford twenty-six known compounds. Their structures were elucidated based on 1D and 2D nuclear magnetic resonance (NMR) spectra and mass spectrometry. High performance liquid chromatography (HPLC) analysis showed that the dominant compounds were 1,2,3,4,6-penta- $O$ galloyl- $\beta$-D-glucopyranoside (16) and 6- $O$ - $m$-digalloyl-1,2,3,4-tetra- $O$-galloyl- $\beta$-D-glucopyranoside (19). The in vitro fungal tyrosinase inhibition potential and the 1,1-diphenyl-2-picrylhydrazyl (DPPH) radical scavenging activity of the extract and isolated compounds were evaluated. $70 \% \mathrm{EtOH}$ extracthas a high tyrosinase inhibitory and good antioxidant activities $\left(\mathrm{IC}_{50}=0.35\right.$ and $2.3 \mu \mathrm{g} \cdot \mathrm{mL}^{-1}$, respectively). Compound 15 (1,2,3,6-tetra-O-galloyl- $\beta$-D-glucopyranoside) showed high tyrosinase inhibitory and antioxidant activities $\left(\mathrm{IC}_{50}=0.23\right.$ and 3.1 $\mu \mathrm{g} \cdot \mathrm{mL}^{-1}$, respectively).
\end{abstract}

Keywords: antioxidant activity, tyrosinase inhibitory activity, Paeoniaceae.

*Address correspondence to this author at the ICMR-UMR CNRS 7312, Campus Sciences, Bât. 18, BP 1039, 51687 Reims, France; Tel/Fax: ++33-326-918208; E-mails: abdulmagid.alabdulmagid@univ-reims.fr.

\section{INTRODUCTION}

Oxidative stress, a deleterious process that can damage all cell structures is generated by reactive oxygen species (ROS) accumulation in the cell, either from excessive production or insufficient neutralization [1,2]. The human body has several mechanisms to counteract oxidative stress by producing antioxidants, which are either naturally produced in situ such as dismutase, peroxidase, and catalase enzymes, as well as glutathione and cytochrome, or externally supplied through foods $[3,4]$. In view of the significant damage occasioned by oxidative stress, in recent years researchers have undertaken the search for antioxidant compounds that can delay or inhibit the initiation or propagation of oxidative chain reaction and thus prevent or repair oxidative damage done to the body's cells by oxygen [5,6]. Currently, a variety of synthetic antioxidant supplements are available. However, antioxidants derived from natural sources have attracted many interests for use in foods or pharmaceutical preparations [7].

Various dermatological disorders such as freckling, age spots and sites of actinic damage are caused by the accumulation of dermal melanin pigment, which is synthesized in melanocytes via the action of tyrosinase, [8-10]. Therefore, there is a large demand for developing antityrosinase and antioxidant products to treat and protect against hyperpigmentation and ageing of the skin caused by ultraviolet rays, ROS, free radicals and other insults.
Paeonia lactiflora Pall. (Paeoniaceae) is an important ornamental and medicinal plant worldwide [11,12]. The roots of $P$. lactiflora are one of the most important sources of bioactive materials in traditional Chinese medicine, with claims of anti-inflammatory, antibacterial, antiviral, antioxidant, antispasmodic, tonic, astringent and analgesic properties [13-15]. The flowers of P. lactiflora are used in aromatherapy. Many papers report the chemical constituents, mainly monoterpenoid glycosides and tannins, in roots of $P$. lactiflora [15-18]. In contrast, the chemical constituents of $P$. lactiflora flowers have not been studied in detail. Recent study indicated that the extract of $P$. lactiflora flowers was rich of polyphenols and eight compounds were identified [19]. A pentagalloylglucose and four flavonoids isolated from the methanol extract of $P$. lactiflora flowers showed significant inhibitory effect on $\mathrm{Cu}^{2+}$-induced low-density lipoprotein [20]. Another study showed that the ethyl ether extract from $P$. lactiflora flowers had antioxidant and antiinflammatory activities [21]. To our knowledge, the antioxidant and tyrosinase inhibitory activities of compounds isolated from $P$. lactiflora flowers have not yet been reported.

Here we report the isolation and structural elucidation of 26 chemical constituents from the ethanol extract of $P$. lactiflora flowers. Antioxidant activities of the isolated compounds were determined by using DPPH scavenging radical assay and their tyrosinase inhibitory effects were evaluated according to in vitro fungal tyrosinase assay. 


\section{MATERIALS AND METHOD}

\subsection{Plant Material and Reagents}

Flowers of Paeonia laciflora Pall. 'Madame de Verneville' were collected in April 2013 by Dr. Laure Pasquier from LVMH research department at Saint Jean de Braye, France. 1,1-diphenyl-2-picrylhydrazyl (DPPH), ascorbic acid, kojic acid, tyrosinase, L-3,4-dihydroxyphenylalanine (L-DOPA), deuterated methanol (MeOD- $\left.d_{4}\right)$ were purchased from Sigma-Aldrich (Saint-Quentin, France). Acetonitrile $\left(\mathrm{CH}_{3} \mathrm{CN}\right)$, methanol $(\mathrm{MeOH})$, trichloromethane $\left(\mathrm{CHCl}_{3}\right)$, ethyl acetate (EtOAc), ethanol (EtOH), dimethyl sulfoxide (DMSO) and trifluoroacetic acid (TFA) were purchased from Carlo Erba Reactifs SDS (Val de Reuil, France).

\subsection{Extraction and Isolation}

The lyophilized flowers of $P$. lactiflora $(50 \mathrm{~g})$ were macerated with EtOH: $\mathrm{H}_{2} \mathrm{O}(7: 3)(1 \mathrm{~L} \mathrm{x} \mathrm{3}$, each 24h) at room temperature. After removal of the solvent, the residue was dissolved in water $(200 \mathrm{~mL})$ and then extracted with EtOAc $(3 \times 100 \mathrm{~mL}$ each $)$ to yield the EtOAc $(8.7 \mathrm{~g})$ and the $\mathrm{H}_{2} \mathrm{O}$ $(19.2 \mathrm{~g})$ extracts. The EtOAc extract was subjected to vacuum liquid chromatography (VLC) over silica gel eluting with $\mathrm{CHCl}_{3}-\mathrm{MeOH}-\mathrm{H}_{2} \mathrm{O}$ (98:2:0-60:40:7) to afford seven fractions EA1-EA7. Fraction EA3 $(0.24 \mathrm{~g})$, eluted with $\mathrm{CHCl}_{3}-\mathrm{MeOH}$ (9:1), was purified by semi-prep HPLC (conditions: $20-25 \%$ of acetonitrile $\left(\mathrm{CH}_{3} \mathrm{CN}\right)$ in $\left.15 \mathrm{~min}\right)$ to afford compounds 7 ( $r t 6.2 \mathrm{~min}, 111 \mathrm{mg}$ ) and 11 (Rt 10.6 min, $49 \mathrm{mg})$. Fraction EA4 (1.44 g), eluted with $\mathrm{CHCl}_{3^{-}}$ $\mathrm{MeOH}(8: 2)$, was purified by preparative HPLC (20-35\% $\mathrm{CH}_{3} \mathrm{CN}$ in $60 \mathrm{~min}$ ) yielding 40 subfractions. Subfraction [21] was purified by semi-prep HPLC (17-21\% of $\mathrm{CH}_{3} \mathrm{CN}$ in 20 $\mathrm{min}$ ) to give compounds 17 (Rt $22.5 \mathrm{~min}, 24 \mathrm{mg}$ ) and 18 (Rt $24.2 \mathrm{~min}, 22 \mathrm{mg}$ ). Subfractions [30-34] was further purified by semi-prep HPLC (isocratic elution with $23 \% \mathrm{CH}_{3} \mathrm{CN}$ ) to afford compounds 25 (Rt $19.2 \mathrm{~min}, 7 \mathrm{mg}$ ) and $\mathbf{2 6}$ (Rt 21.3 min, $23 \mathrm{mg}$ ). Fraction EA5 (2.6 g), eluted with $\mathrm{CHCl}_{3}$ $\mathrm{MeOH}(7: 3)$, was purified by prep. HPLC $\left(20-30 \% \mathrm{CH}_{3} \mathrm{CN}\right.$ in $60 \mathrm{~min}$ ) to give compounds 15 (Rt $20 \mathrm{~min}, 10 \mathrm{mg}$ ), 16 (Rt $23 \mathrm{~min}, 165 \mathrm{mg}$ ), and 19 (Rt $24.2 \mathrm{~min}, 19 \mathrm{mg}$ ). The $\mathrm{H}_{2} \mathrm{O}$ extract (18 g) was subjected to VLC over RP18 $(9 \times 6 \mathrm{~cm})$ eluted with $\mathrm{MeOH}-\mathrm{H}_{2} \mathrm{O}(0: 10-10: 0)$ to give seven fractions (W1-W7). Fractions W2 (0.55 g) and W3 (0.20 g), eluted with $\mathrm{MeOH}-\mathrm{H}_{2} \mathrm{O}$ (1:9), were combined and purified by preparative $\mathrm{HPLC}\left(5-20 \% \mathrm{CH}_{3} \mathrm{CN}\right.$ in $\left.60 \mathrm{~min}\right)$ to give compounds 4 (52 mg) and 2 (12.6 mg). Subfraction [4] was further purified by semi-prep HPLC $\left(0-5 \% \mathrm{CH}_{3} \mathrm{CN}\right.$ in 15 $\mathrm{min}$ ) to afford compounds $\mathbf{3}$ (Rt $5.9 \mathrm{~min}, 4 \mathrm{mg}$ ) and $\mathbf{1}$ (Rt $10.3 \mathrm{~min}, 23 \mathrm{mg}$ ), whereas purification of subfraction [12] in the same conditions yielded compound 5 (Rt $15.3 \mathrm{~min}, 19$ $\mathrm{mg})$. Fraction W4 (0.35 g), eluted with $\mathrm{MeOH}-\mathrm{H}_{2} \mathrm{O}(2: 8)$, was purified by preparative HPLC $\left(5-20 \% \mathrm{CH}_{3} \mathrm{CN}\right.$ in 60 min) to give compound $24(60 \mathrm{mg})$. Subfraction [6] was further purified by semi-prep HPLC (10-17\% $\mathrm{CH}_{3} \mathrm{CN}$ in 20 $\mathrm{min}$ ) to afford compound $\mathbf{9}$ (Rt $11.3 \mathrm{~min}, 17 \mathrm{mg})$. Fraction W5 (1.17 g), eluted with $\mathrm{MeOH}-\mathrm{H}_{2} \mathrm{O}$ (4:6), was purified by preparative $\mathrm{HPLC}\left(20-60 \% \mathrm{CH}_{3} \mathrm{CN}\right.$ in $\left.60 \mathrm{~min}\right)$ to give compound 8 (19 mg). Subfraction [11] was further purified by semi-prep HPLC $\left(10-15 \% \mathrm{CH}_{3} \mathrm{CN}\right.$ in $\left.17 \mathrm{~min}\right)$ to afford compound 13 (Rt $16.3 \mathrm{~min}, 2 \mathrm{mg}$ ). Subfraction [15] was further purified by semi-prep HPLC (isocratic elution $17 \%$ of $\mathrm{CH}_{3} \mathrm{CN}$ ) to afford compounds 22 (Rt $10.3 \mathrm{~min}, 2 \mathrm{mg}$ ) and 10 (Rt $14.2 \mathrm{~min}, 4 \mathrm{mg}$ ). Subfraction [16] was purified by semi-prep HPLC (15-20\% $\mathrm{CH}_{3} \mathrm{CN}$ in $\left.17 \mathrm{~min}\right)$ to afford compound 12 (Rt $13.8 \mathrm{~min}, 12 \mathrm{mg}$ ). Subfractions [20-21] were purified by semi-prep HPLC (15-20\% $\mathrm{CH}_{3} \mathrm{CN}$ in 15 $\mathrm{min}$ ) to afford compound 14 (Rt $13.8 \mathrm{~min}, 35 \mathrm{mg}$ ). Subfractions [24-28] were purified by semi-prep HPLC $\left(19 \% \mathrm{CH}_{3} \mathrm{CN}\right)$ to afford compound 20 (Rt $\left.11.2 \mathrm{~min}, 23 \mathrm{mg}\right)$. Fraction W6 $(0.28 \mathrm{~g})$, eluted with $\mathrm{MeOH}-\mathrm{H}_{2} \mathrm{O}$ (6:4), was purified by prep. HPLC (20-60\% $\mathrm{CH}_{3} \mathrm{CN}$ in $\left.60 \mathrm{~min}\right)$ to give compounds 6 (6 mg), $21(24 \mathrm{mg})$, and $23(6 \mathrm{mg})$. Subfraction [11] was purified by semi-prep HPLC (10-15\% $\mathrm{CH}_{3} \mathrm{CN}$ in 17 $\mathrm{min}$ ) to afford compound 13 (Rt $16.3 \mathrm{~min}, 2 \mathrm{mg}$ ).

\subsection{HPLC Analysis of the 70\% EtOH Extract}

The mobile phase compositions, gradient elution procedure and detection wave length were optimized to get the most useful chemical information and best separation on the HPLC chromatograms of $P$. lactiflora. To improve the resolution and to limit the ionization of target compounds, TFA was added to the binary mixture of $\mathrm{CH}_{3} \mathrm{CN}$-water as recommended by $\mathrm{He}$ et al. [22]. A binary mixture of $\mathrm{CH}_{3} \mathrm{OH}$-water with different concentrations of TFA were also investigated. Finally, the mobile phase consisting of $\mathrm{CH}_{3} \mathrm{CN}-\mathrm{H}_{2} \mathrm{O} / 0.0025 \%$ TFA solution (v/v, $\mathrm{pH} 2.28$ ) was chosen for the determination of $P$. lactiflora fingerprint chromatogram with large number of peaks on the chromatogram achieving within $60 \mathrm{~min}$. The chromatographic separation was achieved using the gradient program: $5 \%$ (for $5 \mathrm{~min}$ ), to $20 \%$ (in $15 \mathrm{~min}$ ), 20\% (for 10 $\mathrm{min}$ ), to $25 \%$ (in $20 \mathrm{~min}$ ) and to $35 \%$ (in $10 \mathrm{~min}$ ) $\mathrm{CH}_{3} \mathrm{CN}$ in $0.0025 \%$ TFA. The chromatogram was monitored at a wavelength of $205 \mathrm{~nm}$ during the experiment. The column temperature was maintained at $30{ }^{\circ} \mathrm{C}$ and the injection volume of sample solution $(5 \mathrm{mg} / \mathrm{mL})$ was $20 \mu \mathrm{L}$. The analysis was performed in triplicates. The $\mathrm{H}_{2} \mathrm{O}$ used for mobile phase was prepared daily, filtered $(0.22 \mu \mathrm{m}$ membrane filter) and then degassed.

\subsection{Tyrosinase Enzyme Assay}

The tyrosinase inhibitory activity was determined according to the method described previously [23]. L-DOPA was used as the substrates in this experiment. Samples were prepared at concentrations of $400,100,50,25$ and $12.5 \mu \mathrm{g} \cdot \mathrm{mL}^{-1}$ in $10 \%$ DMSO in aqueous solution and $100 \mu \mathrm{L}$ of each concentration were added to 96-well plate and then $100 \mu \mathrm{L}$ of $135 \mathrm{U} / \mathrm{mL}$ fungal tyrosinase in phosphate buffer solution (PBS, pH 6.8) were added. After pre-incubation at $25^{\circ} \mathrm{C}$ for $10 \mathrm{~min}, 100 \mu \mathrm{L}$ of L-DOPA $(0.5 \mathrm{mM}$, PBS pH 6.8) were added into 96 -well plate. The reaction mixture was incubated for another $5 \mathrm{~min}$ at $25{ }^{\circ} \mathrm{C}$. The amount of dopachrome in the mixture was determined by the measurement of the absorbance of each well at $475 \mathrm{~nm}$. Kojic acid was used as positive control agent. The inhibitory percentage of tyrosinase was calculated according to the following equation: \% inhibition $=\{[(A-B)-(C-D)] /(A-B)\} \times 100(A$ : $\mathrm{Ab}$ at $475 \mathrm{~nm}$ without test substance; $B: \mathrm{Ab}$ at $475 \mathrm{~nm}$ without test substance and tyrosinase; $C: \mathrm{Ab}$ at $475 \mathrm{~nm}$ with test substance; $D$ : $\mathrm{Ab}$ at $475 \mathrm{~nm}$ with test substance, but without tyrosinase).

\subsection{DPPH Radical Scavenging Activity}

The radical scavenging activity of crude extracts, fractions and purified compounds was measured using the DPPH method [23]. $5 \mu \mathrm{L}$ of different concentrations of the samples were added to $95 \mu \mathrm{L}$ of a DPPH solution $(158 \mu \mathrm{M}$, dissolved 
in EtOH 50\%). The reaction proceeded for $30 \mathrm{~min}$ at $37{ }^{\circ} \mathrm{C}$ on a 96-well microplate and the absorbance was then read at $515 \mathrm{~nm}$. The DPPH inhibition percentage was calculated as followed: $\%$ inhibition $\left[\left(\mathrm{Ab}_{\text {control }}-\mathrm{Ab}_{\text {sample }}\right) / \mathrm{Ab}_{\text {control }}\right] \times 100$. A DPPH solution in EtOH $50 \%$ was used as a control. The curve of the \% scavenging activity against the concentration of sample was prepared by MSExcel based program to obtain the $\mathrm{IC}_{50}$. Samples were prepared at concentrations of $100,50,25,6.2$ and $3.1 \mu \mathrm{g} \cdot \mathrm{mL}^{-1}$. Ascorbic acid was used as a positive control.

\section{EXPERIMENTAL:}

\subsection{General Experimental Procedures}

Absorbance $(\mathrm{Ab})$ values in the DPPH free radical scavenging assay and tyrosinase enzyme assay were read on a Fluostar omega microplate reader (BMG labtech). NMR spectra were carried in $\mathrm{MeOH}-d_{4}$ on Bruker Avance DRX III 500 instruments $\left({ }^{1} \mathrm{H}\right.$ at $500 \mathrm{MHz}$ and ${ }^{13} \mathrm{C}$ at $\left.125 \mathrm{MHz}\right)$. Standard pulse sequences and parameters were used to obtain 1D $\left({ }^{1} \mathrm{H}\right.$ and ${ }^{13} \mathrm{C} J$-mod) and 2D $\left({ }^{1} \mathrm{H}-{ }^{1} \mathrm{H}\right.$ COSY, ROESY, HSQC, and HMBC) spectra. ESI-MS were measured on a Micromass QTOF micro instrument. Silica gel 60 (Merck, 63-200 $\mu \mathrm{m}$ ) or LiChroprep RP18 (Merck, 40-63 $\mu \mathrm{m}$ ) were used for VLC. An Armen instrument equipped with an AP 250/500 pump, ACC 250/500 sampler, and a Merck UV-detector K-2501 was used for preparative HPLC. A Lichrospher RP18 prepacked column (Merck $250 \times 50 \mathrm{~mm}, 12 \mu \mathrm{m}$ ) was used with binary gradient eluent $\left(\mathrm{H}_{2} \mathrm{O}\right.$ and $\left.\mathrm{CH}_{3} \mathrm{CN}\right)$ and a flow rate of $50 \mathrm{~mL} \cdot \mathrm{min}^{-1}$; the chromatogram was monitored at $250 \mathrm{~nm}$. Analytical and semi-preparative HPLC were performed on a Dionex apparatus equipped with an ASI-100 autosampler, an Ultimate 3000 pump, a diode array detector UVD 340S and Chromeleon software. RP18 column (Phenomenex $250 \times 15$ $\mathrm{mm}$, Luna $5 \mu$ ) was used for semi-prep HPLC with binary gradient eluent $\left(\mathrm{H}_{2} \mathrm{O}\left(\mathrm{pH} 2.4\right.\right.$ with TFA); $\left.\mathrm{CH}_{3} \mathrm{CN}\right)$ and a flow rate of $5 \mathrm{~mL} \cdot \mathrm{min}^{-1}$; the chromatogram was monitored at 205 , 210, 250, and $360 \mathrm{~nm}$ whereas an RP18 column (Phenomenex $250 \times 4.6 \mathrm{~mm}$, Luna $5 \mu$ ) was used for analytical HPLC with binary gradient eluent $\left(\mathrm{H}_{2} \mathrm{O}(\mathrm{pH} 2.4\right.$ with TFA); $\mathrm{CH}_{3} \mathrm{CN}$ ) and a flow rate of $1 \mathrm{~mL} \cdot \mathrm{min}^{-1}$.

\subsection{Statistical Analyses}

The data obtained in this study were expressed as mean \pm standard deviation (SD). All the tests were conducted in triplicate and concentrations yielding 50\% inhibition $\left(\mathrm{IC}_{50}\right)$ were determined by interpolation of concentration $\%$ inhibition curve obtained by MSExcel 2010 software.

\section{RESULTS and DISCUSSIONS:}

\subsection{Structure Identification of P. lactiflora Compounds}

Structural elucidation of compounds 1-26 was performed by NMR analysis $\left({ }^{1} \mathrm{H},{ }^{13} \mathrm{C} J\right.$-mod, ${ }^{1} \mathrm{H}-{ }^{1} \mathrm{H}$ COSY, ROESY, HSQC, and HMBC) and mass spectrometry. Their spectroscopic data were in perfect agreement with those reported in the literature.

Compounds isolated from the $\mathrm{H}_{2} \mathrm{O}$ extract

Compound 1: Positive ESI-MS: $m / z$ 193.12 $[\mathrm{M}+\mathrm{Na}]^{+} ;{ }^{1} \mathrm{H}$ NMR: $\delta_{\mathrm{H}} 7.07$ (s, H-2,H-6); ${ }^{13} \mathrm{C}$ NMR : $\delta_{\mathrm{C}} 122.8$ (C-1),
108.8 (C-2,C-6), 144.8 (C-3,C-5), 137.4 (C-4), 170.8 (C-7). Comparing the results with Ref. [24], compound 1 was identified as gallic acid.

Compound 2: Positive ESI-MS: $\mathrm{m} / z$ $517.1[\mathrm{M}+\mathrm{Na}]^{+} ;{ }^{1} \mathrm{H}$ NMR: $\delta_{\mathrm{H}} 3.3-4.10(11 \mathrm{H}, \mathrm{m}$, sugar $\mathrm{H}), 4.41,4.25$ (each $1 \mathrm{H}$, d, $\left.J=12 \mathrm{~Hz}, \mathrm{H}-1^{\prime}\right), 6.95$ (s, H-2",H-6" $) ;{ }^{13} \mathrm{C}$ NMR: $\delta_{\mathrm{C}} 95.5$ (C-1), 72.3 (C-2), 74.0 (C-3), 70.8 (C-4), 74.5 (C-5), 61.8 (C-6), $63.8\left(\mathrm{C}-1^{\prime}\right), 104.4\left(\mathrm{C}-2^{\prime}\right), 78.5\left(\mathrm{C}-3^{\prime}\right), 74.0\left(\mathrm{C}-4^{\prime}\right), 83.0$ (C-5'), 62.2 (C-6'), 119.8 (C-1"), 109.1 (C-2",C-6"), 146.0 (C-3",C-5"),139.1 (C-4"), 166.3 (C-7"). Comparing the results with Ref. [25], compound 2 was identified as $1^{\prime}-O$ galloylsucrose.

Compound 3: Positive ESI-MS: $\mathrm{m} / z$ $517.2[\mathrm{M}+\mathrm{Na}]^{+} ;{ }^{1} \mathrm{H}$ NMR : $\delta_{\mathrm{H}} 3.3-4.2(11 \mathrm{H}, \mathrm{m}$, sugar $\mathrm{H}), 4.4,4.27$ (each $1 \mathrm{H}, \mathrm{m}$, H-6'), 5.42 (d, J=4 Hz, H-1), 7.08 (s, H-2 $\left.{ }^{\prime \prime}, \mathrm{H}-6^{\prime \prime}\right) ;{ }^{13} \mathrm{C}$ NMR: $\delta_{\mathrm{C}} 92.7(\mathrm{C}-1), 72.3(\mathrm{C}-2), 73.8(\mathrm{C}-3), 70.5(\mathrm{C}-4), 75.8$ (C-5), $61.4(\mathrm{C}-6), 62.9\left(\mathrm{C}-1^{\prime}\right), 104.8\left(\mathrm{C}-2^{\prime}\right), 77.9\left(\mathrm{C}-3^{\prime}\right), 75.8\left(\mathrm{C}-4^{\prime}\right)$, $79.9\left(\mathrm{C}-5^{\prime}\right), 66.9\left(\mathrm{C}-6^{\prime}\right), 120.7\left(\mathrm{C}-1^{\prime \prime}\right), 110.2\left(\mathrm{C}-2^{\prime \prime}, \mathrm{C}-6^{\prime \prime}\right)$, 145.8 (C-3",C-5"), 139.2 (C-4"), 168.0 (C-7"). Comparing the results with Ref. [25], compound $\mathbf{3}$ was identified as $6^{\prime}-$ $O$-galloylsucrose.

Compound 4: Positive ESI-MS: $\mathrm{m} / \mathrm{z} 355.3[\mathrm{M}+\mathrm{Na}]^{+} ;{ }^{1} \mathrm{H}$ NMR: $\delta_{\mathrm{H}} 5.52(\mathrm{~d}, J=7.9 \mathrm{~Hz}, \mathrm{H}-1), 3.23(\mathrm{t}, J=8 \mathrm{~Hz}, \mathrm{H}-2), 3.28$ (t, $J=8 \mathrm{~Hz}, \mathrm{H}-3$ ), 3.16 (t, $J=8.2 \mathrm{~Hz}, \mathrm{H}-4), 3.25$ (m, H-5), 3.67 (dd, $J=11.5,2.1, \mathrm{H}-6 \mathrm{a}$ ), 3.47 (dd, $J=11.5,5.1, \mathrm{H}-6 \mathrm{~b}$ ), 5.42 (d, $\left.J=4 \mathrm{~Hz}, \mathrm{H}-1^{\prime}\right), 7.02$ (s, H-2',H-6'); ${ }^{13} \mathrm{C}$ NMR: $\delta_{\mathrm{C}} 94.9(\mathrm{C}-1)$, 73.1 (C-2), 77.1 (C-3), 70.0 (C-4), 78.3 (C-5), 61.0 (C-6), $119.0\left(\mathrm{C}-1^{\prime}\right), 109.4\left(\mathrm{C}-2^{\prime}, \mathrm{C}-6^{\prime}\right), 146.0\left(\mathrm{C}-3^{\prime}, \mathrm{C}-5^{\prime}\right), 139.4$ (C$\left.4^{\prime}\right), 165.1$ (C-7'). Comparing the results with Ref. [26], compound 4 was identified as 1-O-galloyl- $\beta$-Dglucopyranoside.

Compound 5: Positive ESI-MS: $\mathrm{m} / z$, $355.5\lceil\mathrm{M}+\mathrm{Na}\rceil^{+} ;{ }^{1} \mathrm{H}$ NMR: $\delta_{\mathrm{C}} 5.10(\mathrm{~d}, J=3.4 \mathrm{~Hz}, \mathrm{H}-1 \alpha), 4.55(\mathrm{~d}, J=7.9 \mathrm{~Hz}, \mathrm{H}-$ $1 \beta), 4.55(4 \mathrm{H}, \mathrm{m}, \mathrm{H}-6 \alpha \beta), 3.10-4.5(8 \mathrm{H}, \mathrm{m}, \alpha$-glc and $\beta$-glc$\mathrm{H}), 7.10\left(\mathrm{~s}, \mathrm{H}-2^{\prime}, \mathrm{H}-6{ }^{\prime}\right) ;{ }^{13} \mathrm{C}$ NMR : $\delta_{\Gamma} 96.9(\mathrm{C}-1 \beta), 74.8(\mathrm{C}-$

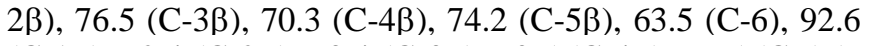
(C-1 $\alpha), 72.4(\mathrm{C}-2 \alpha), 73.4(\mathrm{C}-3 \alpha), 70.5(\mathrm{C}-4 \alpha), 69.5(\mathrm{C}-5 \alpha)$, $120.0\left(\mathrm{C}-1^{\prime}\right), 109.0\left(\mathrm{C}-2^{\prime}, \mathrm{C}-6^{\prime}\right), 145.0\left(\mathrm{C}-3^{\prime}, \mathrm{C}-5^{\prime}\right), 138.4$ (C$\left.4^{\prime}\right), 167.0\left(\mathrm{C}-7^{\prime}\right)$. Comparing the results with Ref. [27], compound $\mathbf{5}$ was identified as 6-O-galloyl-Dglucopyranoside.

Compound 6: Positive ESI-MS: $\mathrm{m} / \mathrm{z} 378.3[\mathrm{M}+\mathrm{Na}]^{+} ;{ }^{1} \mathrm{H}$ NMR: $\delta_{\mathrm{C}} 5.45(\mathrm{~d}, J=7.2 \mathrm{~Hz}, \mathrm{H}-1), 3.25(\mathrm{~m}, \mathrm{H}-2), 3.27(\mathrm{t}$, $J=8.4 \mathrm{~Hz}, \mathrm{H}-3$ ), 3.32 (t, J=8.4 Hz, H-4), 3.29 (m, H-5), 3.55 (m, H-6a), 3.85 (dd, J=11.7, 1.8 Hz, H-6b), 2.31, 2.58 (each d, $\left.J=13.4 \mathrm{~Hz}, \mathrm{H}-2^{\prime}\right), 1.68$ (m, H-4'), 2.24 (m, H-5'), 7.50 (t, $\left.J=6.9 \mathrm{~Hz}, \mathrm{H}-6^{\prime}\right), 1.64\left(\mathrm{~s}, \mathrm{H}-8^{\prime}\right), 1.69\left(\mathrm{~s}, \mathrm{H}-9^{\prime}\right), 1.35$ (s, H10'); ${ }^{13} \mathrm{C}-\mathrm{NMR}: \delta_{\Gamma} 103.7(\mathrm{C}-1), 73.6(\mathrm{C}-2), 77.5(\mathrm{C}-3), 70.1$ (C-4), 77.8 (C-5), 61.2 (C-6), $179.7\left(\mathrm{C}-1^{\prime}\right), 47.2$ (C-2'), 79.6 (C-3'), 23.7 (C-4'), 41.5 (C-5'), 125.3 (C-6'), 138.1 (C-7'), $25.9\left(\mathrm{C}-8^{\prime}\right), 17.9\left(\mathrm{C}-9^{\prime}\right), 23.9\left(\mathrm{C}-10^{\prime}\right)$. Comparing the results with Ref. [28], compound $\mathbf{6}$ was identified as 3-hydroxycitronellic acid 3-O- $\beta$-D-glucopyranoside.

Compound 8: Positive ESI-MS: $m / z 503[\mathrm{M}+\mathrm{Na}]^{+} ;{ }^{1} \mathrm{H}-\mathrm{NMR}$ : $\delta_{\mathrm{H}} 1.84(\mathrm{~d}, J=12.6 \mathrm{~Hz}, \mathrm{H}-3 \mathrm{a}), 2.22$ (d, $\left.J=12.6 \mathrm{~Hz}, \mathrm{H}-3 \mathrm{~b}\right)$, 2.62 (dd, $J=6.6,1.0 \mathrm{~Hz}, \mathrm{H}-5), 1.98$ (dd, $J=11.0,1.0 \mathrm{~Hz}, \mathrm{H}-$ 7a), 2.52 (dd, $J=11.0,6.9 \mathrm{~Hz}, \mathrm{H}-7 \mathrm{~b}), 4.79$ (d, $J=12.6 \mathrm{~Hz}, \mathrm{H}-$ 8a), 4.74 (d, J=12.6 Hz, H-8b), 5.46 ( $s, \mathrm{H}-9$ ), 1.0 (s, H $\mathrm{H}_{3}-10$ ), 
4.55 (d, J=7.6 Hz, H-1'), 3.24 (t, J=8.4 Hz, H-2'), 3.26 (t, $\left.J=8.5 \mathrm{~Hz}, \mathrm{H}-3^{\prime}\right), 3.25$ (m, H-5), 3.65 (dd, $J=11.8,5.1 \mathrm{~Hz}, \mathrm{H}-$ 6'a), 3.88 (dd, $J=11.8,2.1 \mathrm{~Hz}, \mathrm{H}-6^{\prime}$ b), 8.08 (t, $J=8.3 \mathrm{~Hz}, \mathrm{H}-$ 2",H-6"), 7.63 (t, $J=7.5 \mathrm{~Hz}, \mathrm{H}-4 "), 7.50$ (t, $J=7.5 \mathrm{~Hz}, \mathrm{H}-$ $\left.3^{\prime \prime}, \mathrm{H}-5^{\prime \prime}\right) ;{ }^{13} \mathrm{C}-\mathrm{NMR}: \delta_{\mathrm{C}} 98.4(\mathrm{C}-1), 87.3(\mathrm{C}-2), 44.6(\mathrm{C}-3)$, 106.4 (C-4), 44.0 (C-5), 72.3 (C-6), 23.4 (C-7), 61.7 (C-8), 102.3 (C-9), 19.6 (C-10), 100.2 (C-1'), 72.3 (C-2'), 78.1 (C3'), $71.8\left(\mathrm{C}-4^{\prime}\right), 78.0\left(\mathrm{C}-5^{\prime}\right), 62.9\left(\mathrm{C}-6^{\prime}\right), 131.3\left(\mathrm{C}-1^{\prime \prime}\right), 129.7$ (C-2",C-6"), 134.4 (C-4"), 130.8 (C-3",C-5"), 167.8 (C-7"). Comparing the results with Ref. [29], compound 8 was identified as paeoniflorin.

Compound 9 : Positive ESI-MS: $m / z 519.3[\mathrm{M}+\mathrm{Na}]^{+} ;{ }^{1} \mathrm{H}-$ NMR: $\delta_{\mathrm{H}} 1.83$ (d, $\left.J=12.6 \mathrm{~Hz}, \mathrm{H}-3 \mathrm{a}\right), 2.22$ (d, $J=12.6 \mathrm{~Hz}, \mathrm{H}-$ 3b), 2.62 (dd, $J=6.6,1.0 \mathrm{~Hz}, \mathrm{H}-5), 1.99$ (dd, $J=11.1,1.1 \mathrm{~Hz}$, H-7a), 2.51 (dd, $J=11.0,6.9 \mathrm{~Hz}, \mathrm{H}-7 \mathrm{~b}$ ), 4.77 (d, $J=3.5 \mathrm{~Hz}$, $\left.\mathrm{H}_{2}-8\right), 5.45$ (s, H-9), 1.0 (s, $\left.\mathrm{H}_{3}-10\right), 4.55$ (d, $\left.J=7.6 \mathrm{~Hz}, \mathrm{H}-1^{\prime}\right)$, 3.23 (t, $\left.J=8.5 \mathrm{~Hz}, \mathrm{H}-2^{\prime}\right), 3.25$ (t, $\left.J=8.5 \mathrm{~Hz}, \mathrm{H}-3^{\prime}\right), 3.25$ (m, H$\left.5^{\prime}\right), 3.30$ (t, $\left.J=8.4 \mathrm{~Hz}, \mathrm{H}-4^{\prime}\right), 3.66$ (dd, $\left.J=11.9,5.1 \mathrm{~Hz}, \mathrm{H}-6^{\prime} \mathrm{a}\right)$, 3.88 (dd, $\left.J=11.9,2.1 \mathrm{~Hz}, \mathrm{H}-6^{\prime} \mathrm{b}\right), 8.21$ (d, $J=8.8 \mathrm{~Hz}, \mathrm{H}-2^{\prime}$, H$\left.6^{\prime \prime}\right), 7.12\left(\mathrm{~d}, J=8.8 \mathrm{~Hz}, \mathrm{H}-3^{\prime \prime}, \mathrm{H}-5^{\prime \prime}\right) ;{ }^{13} \mathrm{C}-\mathrm{NMR}: \delta_{\mathrm{C}} 98.4(\mathrm{C}-1)$, 87.3 (C-2), 44.6 (C-3), 106.4 (C-4), 44.0 (C-5), 72.3 (C-6), 23.4 (C-7), 61.7 (C-8), 102.3 (C-9), 19.6 (C-10), 100.2 (C1'), 72.3 (C-2'), 78.1 (C-3'), $71.8\left(\mathrm{C}-4^{\prime}\right), 78.0\left(\mathrm{C}-5^{\prime}\right), 62.9$ (C6'), $121.6\left(\mathrm{C}-1^{\prime \prime}\right), 132.4$ (C-2",C-6"), 114.6 (C-3",C-5"), 163.6 (C-4"), 166.9 (C-7"). Comparing the results with Ref. [30], compound 9 was identified as oxypaeoniflorin.

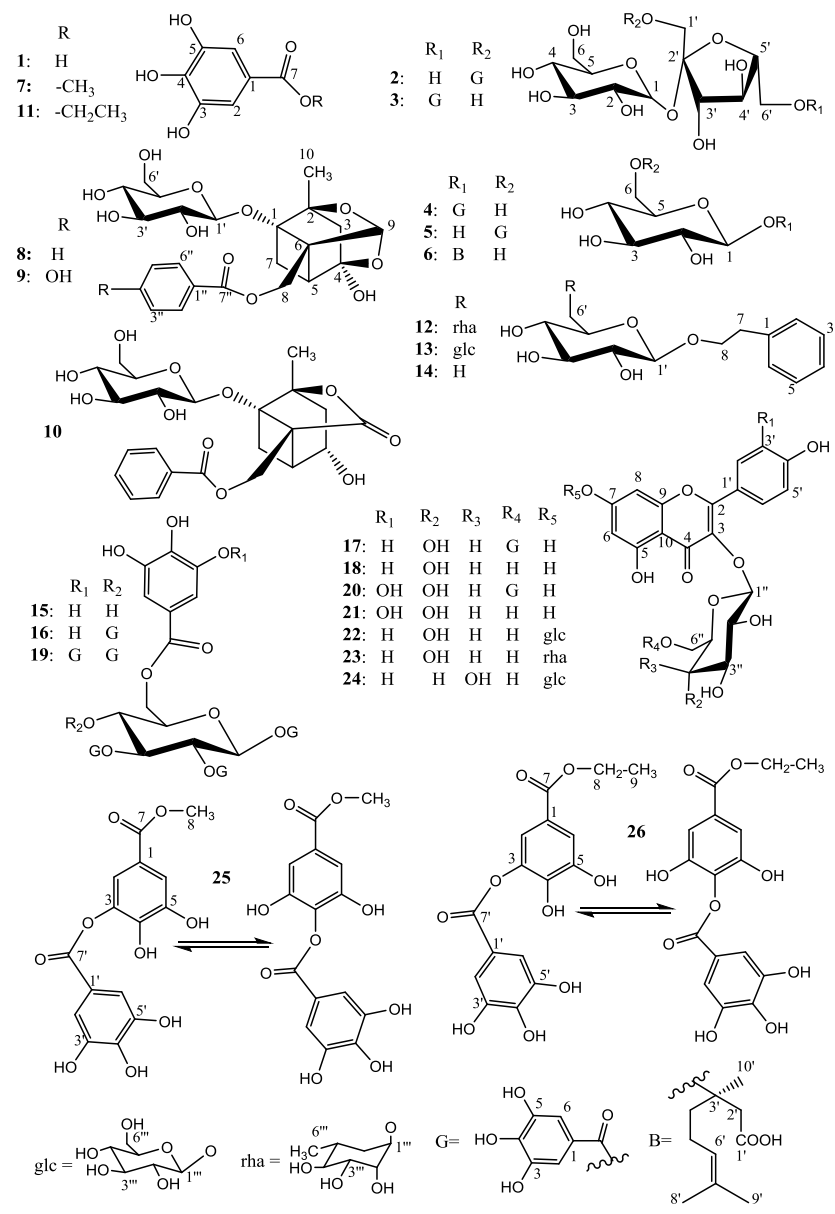

Figure 1: Semi structural formulae of compounds 1-26 isolated from P. lactiflora flowers.
Compound 10: Positive ESI-MS: $\mathrm{m} / z$ $503.6[\mathrm{M}+\mathrm{Na}]^{+}$; ${ }^{1} \mathrm{H}-$ NMR: $\delta_{\mathrm{H}} 2.01$ (dd, $\left.J=15.6,1.6 \mathrm{~Hz}, \mathrm{H}-3 \mathrm{a}\right), 2.40$ (dd, $J=15.6$, $6.8 \mathrm{~Hz}, \mathrm{H}-3 \mathrm{~b}$ ), 4.26 (dd, $J=6.4,4.8 \mathrm{~Hz}, \mathrm{H}-4), 2.91$ (dd, $J=7.5$, $5.1 \mathrm{~Hz}, \mathrm{H}-5), 2.04$ (d, $J=11.2 \mathrm{~Hz}, \mathrm{H}-7 \mathrm{a}), 2.79$ (dd, $J=11.2$, $7.9 \mathrm{~Hz}, \mathrm{H}-7 \mathrm{~b}$ ), 4.67 (d, J=11.2 Hz, H-8a), 4.80 (d, $J=11.2$ $\mathrm{Hz}, \mathrm{H}-8 \mathrm{~b}), 1.52$ (s, $\left.\mathrm{H}_{3}-10\right), 4.52$ (d, $\left.J=7.9 \mathrm{~Hz}, \mathrm{H}-1^{\prime}\right), 3.22$ (t, $\left.J=8.3 \mathrm{~Hz}, \mathrm{H}-2^{\prime}\right), 3.24$ (t, $\left.J=8.4 \mathrm{~Hz}, \mathrm{H}-3^{\prime}\right), 3.35$ (t, $J=8.4 \mathrm{~Hz}$, H-4'), 3.30 (m, H-5'), 3.63 (dd, J=11.7, 4.9 Hz, H-6'a), 3.82 (dd, $J=11.7,2.0 \mathrm{~Hz}, \mathrm{H}-6^{\prime}$ b), 8.07 (dd, $J=8.0,1.0 \mathrm{~Hz}, \mathrm{H}-2^{\prime \prime}$, H-6"), 7.63 (t, $\left.J=7.5 \mathrm{~Hz}, \mathrm{H}-4^{\prime \prime}\right), 7.49$ (t, $J=7.5 \mathrm{~Hz}, \mathrm{H}-3$ ", H$\left.5^{\prime \prime}\right) ;{ }^{13} \mathrm{C}-\mathrm{NMR}: \delta_{\mathrm{C}} 86.9(\mathrm{C}-1), 93.5(\mathrm{C}-2), 41.6(\mathrm{C}-3), 68.4$ (C-4), 41.5 (C-5), 56.9 (C-6), 28.5 (C-7), 62.0 (C-8), 178.0 (C-9), 20.5 (C-10), 100.1 (C-1'), 72.4 (C-2'), 78.1 (C-3'), 71.5 (C-4'), 78.0 (C-5'), 62.8 (C-6'), $131.3\left(\mathrm{C}-1^{\prime \prime}\right), 129.9$ (C2",C-6"), 134.3 (C-4"), 130.7 (C-3",C-5"), 167.9 (C-7"). Comparing the results with Ref. [30], compound 10 was identified as albiflorin.

Compound 12: Positive ESI-MS: $m / z$ 441.5 $[\mathrm{M}+\mathrm{Na}]^{+} ;{ }^{1} \mathrm{H}-$ NMR: $\delta_{\mathrm{H}} 7.09$ (d, $\left.J=7.4 \mathrm{~Hz}, \mathrm{H}-2, \mathrm{H}-6\right), 7.50(\mathrm{t}, J=7.4 \mathrm{~Hz}, \mathrm{H}-$ 3, H-5), 7.62 (t, $J=7.4 \mathrm{~Hz}, \mathrm{H}-4), 2.97$ (dt, $J=7.2,4.2 \mathrm{~Hz}, \mathrm{H}-7$ ), 3.78 (dq, $J=9.7,7.2 \mathrm{~Hz}, \mathrm{H}-8 \mathrm{a}), 4.06(\mathrm{dq}, J=9.6,7.2 \mathrm{~Hz}, \mathrm{H}-$ 8b), 4.32 (d, $J=7.8 \mathrm{~Hz}, \mathrm{H}-1^{\prime}$ ), 3.20 (t, $\left.J=8.8 \mathrm{~Hz}, \mathrm{H}-2^{\prime}\right), 3.36$ (t, $\left.J=8.4 \mathrm{~Hz}, \mathrm{H}-3^{\prime}\right), 3.30$ (t, $\left.J=8.4 \mathrm{~Hz}, \mathrm{H}-4^{\prime}\right), 3.41$ (m, H-5'), 3.64 (dd, $J=11.2,5.8 \mathrm{~Hz}, \mathrm{H}-6$ 'a), 4.00 (dd, $J=11.2,1.1 \mathrm{~Hz}$, H-6'b), 4.70 (s, H-1"'), 3.86 (dd, J=3.2, $1.1 \mathrm{~Hz}, \mathrm{H}-2^{\prime \prime}$ ), 3.70 (dd, $\left.J=8.4,3.3 \mathrm{~Hz}, \mathrm{H}-3^{\prime \prime}\right), 3.39$ (t, $\left.J=8.5 \mathrm{~Hz}, \mathrm{H}-4^{\prime \prime}\right), 3.70$ (m, H-5"), 1.28 (d, J=6.2 Hz, H-6"'); ${ }^{13} \mathrm{C}-\mathrm{NMR}: \delta_{\mathrm{C}} 140.1(\mathrm{C}-1)$, 130.1 (C-2,C-6), 127.2 (C-4), 139.4 (C-3,C-5), 37.3 (C-7), 72.2 (C-8), $104.5\left(\mathrm{C}-1^{\prime}\right), 75.0\left(\mathrm{C}-2^{\prime}\right), 78.1\left(\mathrm{C}-3^{\prime}\right), 71.4\left(\mathrm{C}-4^{\prime}\right)$, $77.2\left(\mathrm{C}-5^{\prime}\right), 68.1$ (C-6'), 102.2 (C-1"), 72.1 (C-2"), 72.3 (C$\left.3^{\prime \prime}\right), 74.1\left(\mathrm{C}-4^{\prime \prime}\right), 69.8\left(\mathrm{C}-5^{\prime \prime}\right), 18.0\left(\mathrm{C}-6^{\prime \prime}\right)$. Comparing the results with Ref. [31], compound $\mathbf{1 2}$ was identified as 2phenylethyl-[ $\alpha$-L-rhamnopyranosyl-( $1 \rightarrow 6)]-\beta$-Dglucopyranoside.

Compound 13: Positive ESI-MS: $\mathrm{m} / z$ $457.5[\mathrm{M}+\mathrm{Na}]^{+} ;{ }^{1} \mathrm{H}-$ NMR: $\delta_{\mathrm{H}} 7.30(\mathrm{~m}, \mathrm{H}-2-\mathrm{H}-6), 2.95(\mathrm{t}, J=7.2 \mathrm{~Hz}, \mathrm{H}-7), 3.79$ (dq, $J=9.7,7.8 \mathrm{~Hz}, \mathrm{H}-8 \mathrm{a}), 4.10$ (dq, $J=9.6,7.7 \mathrm{~Hz}, \mathrm{H}-8 \mathrm{~b}$ ), 4.34 (d, J=7.8 Hz, H-1'), 3.22 (t, J=8.3 Hz, H-2'), 3.39 (t, $J=8.4 \mathrm{~Hz}, \mathrm{H}-3^{\prime}$ ), 3.32 (t, $J=8.4 \mathrm{~Hz}, \mathrm{H}-4^{\prime}$ ), 3.49 (m, H-5'), 3.81 (dd, $J=11.7,5.3 \mathrm{~Hz}, \mathrm{H}-6$ 'a), 4.18 (dd, $J=11.7,1.8 \mathrm{~Hz}$, H-6'b), 4.40 (d, $\left.J=7.8 \mathrm{~Hz}, \mathrm{H}-1^{\prime \prime}\right), 3.22$ (t, $\left.J=8.2 \mathrm{~Hz}, \mathrm{H}-2^{\prime \prime}\right)$, 3.37 (t, $\left.J=8.4 \mathrm{~Hz}, \mathrm{H}-3^{\prime \prime}\right), 3.30$ (t, $\left.J=8.5 \mathrm{~Hz}, \mathrm{H}-4^{\prime \prime}\right), 3.29$ (m, H-5"), 3.68 (dd, $\left.J=11.9,5.1 \mathrm{~Hz}, \mathrm{H}-6^{\prime \prime} \mathrm{a}\right), 3.88$ (dd, $J=11.9$, $\left.1.5 \mathrm{~Hz}, \mathrm{H}-6{ }^{\prime \prime} \mathrm{b}\right) ;{ }^{13} \mathrm{C}-\mathrm{NMR}: \delta_{\mathrm{C}} 140.1$ (C-1), 130.1 (C-2, C-6), 127.2 (C-4), 139.4 (C-3, C-5), 37.2 (C-7), 71.9 (C-8), 104.4 (C-1'), 75.0 (C-2'), $78.0\left(\mathrm{C}-3^{\prime}\right), 71.4\left(\mathrm{C}-4^{\prime}\right), 77.2\left(\mathrm{C}-5^{\prime}\right), 69.8$ (C-6'), 104.8 (C-1"), 75.1 (C-2"), $78.1\left(\mathrm{C}-3^{\prime \prime}\right), 71.6$ (C-4"), 77.9 (C-5"), $62.7\left(\mathrm{C}-6^{\prime \prime}\right)$. Comparing the results with Ref. [32], compound $\mathbf{1 3}$ was identified as 2-phenylethyl)]- $\beta$-Dglucopyranosyl-( $1 \rightarrow 6)]$ - $\beta$-D-glucopyranoside.

Compound 14: Positive ESI-MS: $\mathrm{m} / z 307.6[\mathrm{M}+\mathrm{Na}]^{+} ;{ }^{1} \mathrm{H}-$ NMR: $\delta_{\mathrm{H}} 7.30(\mathrm{~m}, \mathrm{H}-2-\mathrm{H}-6), 2.95(\mathrm{td}, J=7.1,1.5 \mathrm{~Hz}, \mathrm{H}-7)$, 3.79 (dq, $J=9.6,7.2 \mathrm{~Hz}, \mathrm{H}-8 \mathrm{a}), 4.11(\mathrm{dq}, J=9.6,7.7 \mathrm{~Hz}, \mathrm{H}-$ 8b), 4.32 (d, $J=7.8 \mathrm{~Hz}, \mathrm{H}-1^{\prime}$ ), 3.22 (t, $J=8.4 \mathrm{~Hz}, \mathrm{H}-2^{\prime}$ ), 3.38 (t, $\left.J=8.4 \mathrm{~Hz}, \mathrm{H}-3^{\prime}\right), 3.35$ (t, $\left.J=8.4 \mathrm{~Hz}, \mathrm{H}-4^{\prime}\right), 3.32$ (m, H-5'), 3.71 (dd, $J=11.7,4.9 \mathrm{~Hz}, \mathrm{H}-6$ 'a), 3.88 (dd, $J=11.7,2.0 \mathrm{~Hz}$, H-6'b); ${ }^{13} \mathrm{C}-\mathrm{NMR}: \delta_{\mathrm{C}} 140.0$ (C-1), 129.3 (C-2, C-6), 127.2 (C-4), 130.0 (C-3, C-5), 37.2 (C-7), 71.7 (C-8), 104.3 (C-1'), 75.1 (C-2'), 78.1 (C-3'), 71.6 (C-4'), 77.9 (C-5'), 62.7 (C-6'). Comparing the results with Ref. [19], compound 14 was identified as 2-phenylethyl- $\beta$-D-glucopyranoside. 
Compound 20: Positive ESI-MS: $\mathrm{m} / \mathrm{z} 639.0[\mathrm{M}+\mathrm{Na}]^{+} ;{ }^{1} \mathrm{H}$ NMR: $\delta_{\mathrm{H}} 6.20(\mathrm{~d}, J=2.0 \mathrm{~Hz}, \mathrm{H}-6), 6.38(\mathrm{~d}, J=2.0 \mathrm{~Hz}, \mathrm{H}-8)$, 7.55 (d, $\left.J=2.1 \mathrm{~Hz}, \mathrm{H}-2^{\prime}\right), 6.73$ (d, $\left.J=8.5 \mathrm{~Hz}, \mathrm{H}-5^{\prime}\right), 7.59$ (dd, $\left.J=8.8,2.1 \mathrm{~Hz}, \mathrm{H}-6^{\prime}\right), 5.20$ (d, $\left.J=7.5 \mathrm{~Hz}, \mathrm{H}-1^{\prime \prime}\right), 3.50$ (t, $J=8.5$ $\left.\mathrm{Hz}, \mathrm{H}-2^{\prime \prime}\right), 3.48$ (t, $\left.J=8.5 \mathrm{~Hz}, \mathrm{H}-3^{\prime \prime}\right), 3.36$ (t, $\left.J=9 \mathrm{~Hz}, \mathrm{H}-4^{\prime \prime}\right)$, 3.25 (m, H-5"), 4.30 (dd, J=11.5, $\left.1.9 \mathrm{~Hz}, \mathrm{H}-6^{\prime \prime} \mathrm{a}\right), 4.36$ (dd, $\left.J=11.5,5.0 \mathrm{~Hz}, \mathrm{H}-6^{\prime \prime} \mathrm{b}\right) ; 6.95$ (s, H-2"',H-6"' $) ;{ }^{13} \mathrm{C}$ NMR: $\delta_{\mathrm{C}}$ 158.0 (C-2), 133.7 (C-3), 177.8 (C-4), 161.6 (C-5), 99.1 (C6), 164.6 (C-7), 93.9 (C-8), 156.7 (C-9), 104.3 (C-10), 121.3 (C-1'), 115.7 (C-2'), 145.2 (C-3'), 148.8 (C-4'), 116.1 (C-5'), $121.3\left(\mathrm{C}-6^{\prime}\right), 102.8\left(\mathrm{C}-1^{\prime \prime}\right), 74.4\left(\mathrm{C}-2^{\prime \prime}\right), 76.6$ (C-3"), 70.1 (C4"), $75.6\left(\mathrm{C}-5^{\prime \prime}\right), 62.9$ (C-6") 119.8 (C-1"'), 109.1 (C-2"',C6"'), 145.8 (C-3"',C-5"'), 138.8 (C-4"'), 166.8 (C-7"'). Comparing the results with Ref. [33], compound 20 was identified as quercetin-3-O-(6"-O-galloyl)-glucoside.

Compound 21: ESI-MS: $m / z 487.2[\mathrm{M}+\mathrm{Na}]^{+} ;{ }^{1} \mathrm{H}$ NMR: $\delta_{\mathrm{H}}$ 6.62 (d, $J=1.8 \mathrm{~Hz}, \mathrm{H}-6), 6.40$ (d, $J=1.8 \mathrm{~Hz}, \mathrm{H}-8), 7.75$ (d, $\left.J=1.5 \mathrm{~Hz}, \mathrm{H}-2^{\prime}\right), 6.90$ (d, $\left.J=8.4, \mathrm{~Hz}, \mathrm{H}-5^{\prime}\right), 7.60$ (dd, $J=8.4$, $\left.1.5 \mathrm{~Hz}, \mathrm{H}-6^{\prime}\right), 5.25$ (d, $\left.J=7.4 \mathrm{~Hz}, \mathrm{H}-1^{\prime \prime}\right), 3.52$ (t, $J=8.5 \mathrm{~Hz}, \mathrm{H}-$ $\left.2^{\prime \prime}\right), 3.45$ (t, $\left.J=8.5 \mathrm{~Hz}, \mathrm{H}-3^{\prime \prime}\right), 3.40$ (t, $\left.J=8.5 \mathrm{~Hz}, \mathrm{H}-4^{\prime \prime}\right), 3.25$ (m, H-5"), 3.61 (dd, J=11.8, 5.3 Hz, H-6"a), 3.74 (dd, $\left.J=11.8,2.0 \mathrm{~Hz}, \mathrm{H}-6^{\prime \prime} \mathrm{b}\right) ;{ }^{13} \mathrm{C}$ NMR: $\delta_{\mathrm{C}} 158.4(\mathrm{C}-2), 133.6(\mathrm{C}-$ 3), 179.4 (C-4), 162.9 (C-5), 100.1 (C-6), 166.5 (C-7), 94.8 (C-8), 158.9 (C-9), 105.5 (C-10), 123.0 (C-1'), 116.0 (C-2'), 145.9 (C-3'), 149.8 (C-4'), $117.6\left(\mathrm{C}-5^{\prime}\right), 123.3\left(\mathrm{C}-6^{\prime}\right), 104.4$ $\left(\mathrm{C}-1^{\prime \prime}\right), 75.7$ (C-2"), 78.3 (C-3"), 71.1 (C-4"), 78.6 (C-5"), $62.5\left(\mathrm{C}-6^{\prime \prime}\right)$. Comparing the results with reference data [19], compound 21was identified as quercetin 3-O- $\beta$-Dglucopyranoside.

Compound 22: positive ESI-MS: $m / z 633.2[\mathrm{M}+\mathrm{Na}]^{+} ;{ }^{1} \mathrm{H}$ NMR: $\delta_{\mathrm{H}} 6.55(\mathrm{~d}, J=2.0 \mathrm{~Hz}, \mathrm{H}-6), 6.80(\mathrm{~d}, J=2.0 \mathrm{~Hz}, \mathrm{H}-8)$, 8.11 (d, $\left.J=8.0 \mathrm{~Hz}, \mathrm{H}-2^{\prime}, \mathrm{H}^{\prime} 6^{\prime}\right), 6.91$ (d, $J=9.0 \mathrm{~Hz}, \mathrm{H}-3^{\prime}, \mathrm{H}-5^{\prime}$ ), 5.35 (d, J=7.9 Hz, H-1"), 3.46 (t, J=8.1 Hz, H-2"), 3.32 (t, $\left.J=8.5 \mathrm{~Hz}, \mathrm{H}-3^{\prime \prime}\right), 3.32\left(\mathrm{~m}, \mathrm{H}-4^{\prime \prime}\right), 3.45$ (m, H-5"), 3.55 (dd, $\left.J=11.8,5.2 \mathrm{~Hz}, \mathrm{H}-6^{\prime \prime} \mathrm{a}\right), 3.73$ (dd, $J=11.8,2.1 \mathrm{~Hz}, \mathrm{H}-6^{\prime \prime} \mathrm{b}$ ), 5.08 (d, J=7.7 Hz, H-1'"'), 3.53 (m, H-2'"'), 3.52 (m, H-3"'), 3.42 (t, $\left.J=8.4 \mathrm{~Hz}, \mathrm{H}-4^{\prime \prime \prime}\right), 3.24$ (m, H-5"')), 3.75 (dd, $J=11.9$, $\left.5.1 \mathrm{~Hz}, \mathrm{H}-66^{\prime \prime \prime} \mathrm{a}\right), 3.97$ (dd, $\left.J=11.9,2.1 \mathrm{~Hz}, \mathrm{H}-6{ }^{\prime \prime \prime} \mathrm{b}\right) ;{ }^{13} \mathrm{C}$ NMR: $\delta_{\mathrm{C}} 155.5$ (C-2), 134.1 (C-3), 178.1 (C-4), 161.4 (C-5), 100.3 (C-6), 163.2 (C-7), 94.7 (C-8), 156.3 (C-9), 105.5 (C10), 121.3 (C-1'), 131.0 (C-2', C-6'), 114.7 (C-3',C-5'), 160.3 (C-4'), 102.3 (C-1"), 74.3 (C-2"), $76.4\left(\mathrm{C}-3^{\prime \prime}\right), 70.0$ (C-4"), 76.9 (C-5"), 61.2 (C-6"), 100.2 (C-1"'), 73.3 (C-2'"'), 77.2 (C-3"'), 69.9 (C-4"'), 76.8 (C-5'"'), 62.2 (C-6"'). Comparing the results with reference data [34], compound 22 was identified as kaempferol- 3, 7-di-O- $\beta$-D-glucopyranoside.

Compound 23: Positive ESI-MS: $\mathrm{m} / \mathrm{z} 617.3[\mathrm{M}+\mathrm{Na}]^{+} ;{ }^{1} \mathrm{H}-$ NMR: $\delta_{\mathrm{H}} 6.50$ (d, $\left.J=1.7 \mathrm{~Hz}, \mathrm{H}-6\right), 6.79$ (d, $\left.J=1.7 \mathrm{~Hz}, \mathrm{H}-8\right)$, 8.11 (d, J=8.4 Hz, H-2', H-6'), 6.92 (d, J=8.4 Hz, H-3', H-5'), 5.33 (d, $\left.J=7.2 \mathrm{~Hz}, \mathrm{H}-1^{\prime \prime}\right), 3.46$ (t, $\left.J=8.2 \mathrm{~Hz}, \mathrm{H}-2^{\prime \prime}\right), 3.44$ (t, $\left.J=8.2 \mathrm{~Hz}, \mathrm{H}-3^{\prime \prime}\right), 3.33$ (m, H-4"), 3.35 (m, H-5"), 3.55 (dd, $J=11.9,5.0 \mathrm{~Hz}, \mathrm{H}-6^{\prime \prime}$ a), 3.72 (dd, $J=11.9,1.9 \mathrm{~Hz}, \mathrm{H}-6^{\prime \prime}$ ) ), 5.60 (s, H-1"'), 4.05 (dd, J=3.3, $\left.1.0 \mathrm{~Hz}, \mathrm{H}-2^{\prime \prime \prime}\right), 3.86$ (dd, $\left.J=8.3,3.3 \mathrm{~Hz}, \mathrm{H}-3^{\prime \prime \prime}\right), 3.50$ (t, $\left.J=8.5 \mathrm{~Hz}, \mathrm{H}-4^{\prime \prime \prime}\right), 3.61$ (m, H$\left.5^{\prime \prime \prime}\right), 1.17\left(\mathrm{~d}, J=6.2 \mathrm{~Hz}, \mathrm{H}-6^{\prime \prime \prime}\right) ;{ }^{13} \mathrm{C}$ NMR: $\delta_{\mathrm{C}} 159.6(\mathrm{C}-2)$, 135.6 (C-3), 179.7 (C-4), 161.9 (C-5), 100.6 (C-6), 163.6 (C7), 95.5 (C-8), 158.1 (C-9), 107.4 (C-10), 122.6 (C-1'), 132.4 (C-2',C-6'), 116.2 (C-3',C-5'), 161.8 (C-4'), 103.7 (C-1"), $75.7\left(\mathrm{C}-2^{\prime \prime}\right), 78.1\left(\mathrm{C}-3^{\prime \prime}\right), 71.4\left(\mathrm{C}-4^{\prime \prime}\right), 77.5\left(\mathrm{C}-5^{\prime \prime}\right), 62.6(\mathrm{C}-$ 6"), 99.8 (C-1"'), 71.7 (C-2'"'), 72.2 (C-3'"'), 73.5 (C-4"'),
71.2 (C-5"'), 18.1 (C-6"'). Comparing the results with reference data [35], compound $\mathbf{2 3}$ was identified as kaempferol rhamnopyranoside.

Compound 24: positive ESI-MS: $\mathrm{m} / z 633.1[\mathrm{M}+\mathrm{Na}]^{+} ;{ }^{1} \mathrm{H}$ NMR: $\delta_{\mathrm{H}} 6.47(\mathrm{~d}, J=2.0 \mathrm{~Hz}, \mathrm{H}-6), 6.81(\mathrm{~d}, J=2.0 \mathrm{~Hz}, \mathrm{H}-8)$, 8.09 (d, $\left.J=8.9 \mathrm{~Hz}, \mathrm{H}-2^{\prime}, \mathrm{H}-6^{\prime}\right), 6.91$ (d, $J=8.9 \mathrm{~Hz}, \mathrm{H}-3^{\prime}, \mathrm{H}-5^{\prime}$ ), 5.50 (d, $\left.J=7.4 \mathrm{~Hz}, \mathrm{H}-1^{\prime \prime}\right), 3.20$ (t, $\left.J=8.0 \mathrm{~Hz}, \mathrm{H}-2^{\prime \prime}\right), 3.25$ (dd, $\left.J=8.0,3.3 \mathrm{~Hz}, \mathrm{H}-3^{\prime \prime}\right), 3.09$ (d, J=3.3, H-4"'), 3.11 (m, H-5"), 3.35 (m, H-6"a), 3.57 (dd, J=11.8, 1.1 Hz, H-6"b), 5.10 (d, $\left.J=7.4 \mathrm{~Hz}, \mathrm{H}-1^{\prime \prime \prime}\right), 3.27$ (m, H-2"'), 3.34 (t, $\left.J=8.0 \mathrm{~Hz}, \mathrm{H}-3^{\prime \prime \prime}\right)$, 3.20 (t, J=8.4 Hz, H-4"'), 3.46 (m, H-5'"'), 3.47 (m, H-6"'a), 3.71 (dd, $\left.J=11.8,1.1 \mathrm{~Hz}, \mathrm{H}-66^{\prime \prime \prime} \mathrm{b}\right) ;{ }^{13} \mathrm{C}$ NMR: $\delta_{\mathrm{C}} 157.3(\mathrm{C}-2)$, 133.9 (C-3), 178.1 (C-4), 161.3 (C-5), 99.8 (C-6), 163.3 (C7), 94.9 (C-8), 156.5 (C-9), 106.1 (C-10), 121.2 (C-1'), 131.5 (C-2',C-6'), $115.6\left(\mathrm{C}-3^{\prime}, \mathrm{C}-5^{\prime}\right), 160.7$ (C-4'), $101.3\left(\mathrm{C}-1^{\prime \prime}\right)$, 74.7 (C-2"), 76.9 (C-3"), 70.3 (C-4"), 78.0 (C-5"), 61.3 (C$\left.6^{\prime \prime}\right), 101.2\left(\mathrm{C}-1^{\prime \prime \prime}\right), 73.6\left(\mathrm{C}-2^{\prime \prime \prime}\right), 77.0\left(\mathrm{C}-3^{\prime \prime \prime}\right), 70.1$ (C-4"'), $77.6\left(\mathrm{C}-5^{\prime \prime \prime}\right), 62.1$ (C-6"'). Comparing the results with reference data [33], compound $\mathbf{2 4}$ was identified as kaempferol 3-O- $\beta$-D-galactopyranosyl-7- $O-\beta$-Dglucopyranoside.

\section{Compounds isolated from the EtOAc extract}

Compound 7: Positive ESI-MS: $\mathrm{m} / z$ 207.5 $\left[\mathrm{M}+\left.\mathrm{Na}\right|^{+} ;{ }^{1} \mathrm{H}\right.$ NMR: $\delta_{\mathrm{H}} 7.08\left(2 \mathrm{H}, \mathrm{s}, \mathrm{H}-2^{\prime}, \mathrm{H}-6^{\prime}\right), 3.82\left(3 \mathrm{H}, \mathrm{s}, \mathrm{OCH}_{3}\right),{ }^{13} \mathrm{C}$ NMR: $\delta_{\mathrm{C}} 120.3$ (C-1), 109.3 (C-2, C-6), 144.8 (C-3,C-5), 138.2 (C-4), $168.0(\mathrm{C}-7), 52.3\left(\mathrm{OCH}_{3}\right)$. Comparing the results with Ref. [32], compound 7 was identified as methyl gallate.

Compound 11: Positive ESI-MS: $m / z, 221.4\left[\mathrm{M}+\left.\mathrm{Na}\right|^{+}{ }^{1} \mathrm{H}\right.$ NMR $\left(500 \mathrm{MHz}, \mathrm{MeOH}-d_{4}\right) \delta: 7.10\left(2 \mathrm{H}, \mathrm{s}, \mathrm{H}-2^{\prime}, \mathrm{H}-6^{\prime}\right), 3.82$ $\left(3 \mathrm{H}, \mathrm{s}, \mathrm{OCH}_{3}\right), 1.40\left(3 \mathrm{H}, \mathrm{t}, J=7.1 \mathrm{~Hz}, \mathrm{CH}_{3}\right), 4.35(2 \mathrm{H}, \mathrm{q}$, $\left.J=7.1 \mathrm{~Hz}, \mathrm{OCH}_{2}\right) ;{ }^{13} \mathrm{C}$ NMR $\left(125 \mathrm{MHz}, \mathrm{MeOH}-d_{4}\right) \delta: 121.8$ (C-1), 110.0 (C-2, C-6), 146.5 (C-3,C-5), 139.7 (C-4), 168.5 (C-7), $14.6\left(\mathrm{CH}_{3}\right), 61.7\left(\mathrm{OCH}_{2}\right)$. Comparing the results with Ref. [32], compound $\mathbf{1 1}$ was identified as ethyl gallate.

Compound 15: Positive ESI-MS: $m / z 811.5[\mathrm{M}+\mathrm{Na}]^{+} ;{ }^{1} \mathrm{H}$ NMR: $\delta_{\mathrm{H}} 6.24(\mathrm{~d}, J=8.0 \mathrm{~Hz}, \mathrm{H}-1), 5.48(\mathrm{t}, J=8.3 \mathrm{~Hz}, \mathrm{H}-2)$, $5.52(\mathrm{t}, J=8.5 \mathrm{~Hz}, \mathrm{H}-3), 3.90$ (t, $J=8.5 \mathrm{~Hz}, \mathrm{H}-4), 4.10$ (m, H5), 4.52 (m, H-6a), 4.60 (dd, J=12.0, $1.0 \mathrm{~Hz}, \mathrm{H}-6 \mathrm{~b}), 7.60$, 7.20, 7.01, 6.98 (each s, galloyl H-2', H-6'); ${ }^{13} \mathrm{C}$ NMR: $\delta_{\mathrm{C}}$ 93.6 (C-1), 71.9 (C-2), 76.1 (C-3), 69.5 (C-4), 76.1 (C-5), 63.4 (C-6), 118.0, 118.1, 119.0, $119.2\left(4 \times \mathrm{C}^{\prime} \mathbf{1}^{\prime}\right), 107.9$, 109.0, 109.2, $109.2\left(4 \times \mathrm{C}-2^{\prime}, 4 \times \mathrm{C}-6^{\prime}\right), 144.9,145.0,145.1$, $145.3\left(4 \times \mathrm{C}^{\prime} 3^{\prime}, 4 \times \mathrm{C}-5^{\prime}\right), 138.2,138.5,138.9,139.1(4 \times \mathrm{C}-$ $\left.4^{\prime}\right), 164.8,165.1,165.5,166.5\left(4 \times \mathrm{C}-7^{\prime}\right)$. Comparing the results with Ref. [36], compound $\mathbf{1 5}$ was identified as 1,2,3,6-tetra- $O$-galloyl- $\beta$-D-glucopyranoside.

Compound 16: Positive ESI-MS: $m / z 963.5[\mathrm{M}+\mathrm{Na}]^{+} ;{ }^{1} \mathrm{H}$ NMR: $\delta_{\mathrm{H}} 6.28(\mathrm{~d}, J=7.9 \mathrm{~Hz}, \mathrm{H}-1), 5.61(\mathrm{t}, J=8.4 \mathrm{~Hz}, \mathrm{H}-2)$, $5.93(\mathrm{t}, J=8.5 \mathrm{~Hz}, \mathrm{H}-3), 5.65$ (t, $J=8.5 \mathrm{~Hz}, \mathrm{H}-4), 4.42(\mathrm{~m}, \mathrm{H}-$ 5), 4.41 (m, H-6a), 4.55 (dd, J=12.0, $1.0 \mathrm{~Hz}, \mathrm{H}-6 \mathrm{~b}), 7.14$, 7.07, 7.01, 6.98, 6.87 (each s, galloyl H-2', H-6'); ${ }^{13} \mathrm{C}$ NMR: $\delta_{\mathrm{C}} 92.4(\mathrm{C}-1), 70.8(\mathrm{C}-2), 72.7(\mathrm{C}-3), 68.4(\mathrm{C}-4), 73.0(\mathrm{C}-5)$, 61.7 (C-6), 118.3, 118.8, 118.9, 119.0, $119.4\left(5 \times \mathrm{C}-1^{\prime}\right)$, $108.9,109.0,109.1,109.2,109.3\left(5 \times \mathrm{C}-2^{\prime}, 5 \times \mathrm{C}-6^{\prime}\right), 144.9$, $145.0,145.1,145.2,145.3\left(5 \times \mathrm{C}^{-} 3^{\prime}, 5 \times \mathrm{C}-5^{\prime}\right), 138.6,138.7$, $138.8,138.9,139.4\left(5 \times \mathrm{C}-4^{\prime}\right), 164.8,165.5,165.6,165.9$, $166.5\left(5 \times C-7^{\prime}\right)$. Comparing the results with Ref. [37], 
compound 16 was identified as 1,2,3,4,6-penta-O-galloyl- $\beta$ D-glucopyranoside.

Compound 17: ESI-MS: $m / z 623.0[\mathrm{M}+\mathrm{H}]^{+} ;{ }^{1} \mathrm{H}$ NMR: $\delta_{\mathrm{H}}$ 6.18 (d, $J=2.0 \mathrm{~Hz}, \mathrm{H}-6), 6.39$ (d, $J=2.0 \mathrm{~Hz}, \mathrm{H}-8), 7.92$ (d, $\left.J=8.4 \mathrm{~Hz}, \mathrm{H}-2^{\prime}, \mathrm{H}-6^{\prime}\right), 6.76$ (d, $\left.J=8.4 \mathrm{~Hz}, \mathrm{H}-3^{\prime}, \mathrm{H}-5^{\prime}\right), 5.42$ (d, $\left.J=7.2 \mathrm{~Hz}, \mathrm{H}-1^{\prime \prime}\right), 4.17\left(\mathrm{dd}, J=2.0 \mathrm{~Hz}, \mathrm{H}-6^{\prime \prime}\right), 3.51$ (t, $J=8.4$ $\left.\mathrm{Hz}, \mathrm{H}-2^{\prime \prime}\right), 3.49$ (t, $\left.J=8.4 \mathrm{~Hz}, \mathrm{H}-3^{\prime \prime}\right), 3.34$ (t, $J=9.1 \mathrm{~Hz}, \mathrm{H}-$ $\left.4^{\prime \prime}\right), 3.24$ (m, H-5"), 4.32 (dd, $\left.J=11.8,1.8 \mathrm{~Hz}, \mathrm{H}-6^{\prime \prime} \mathrm{a}\right), 4.34$ (dd, J=11.8, 5.2 Hz, H-6"'b), 6.89 (s, H-2"', H-6"'); ${ }^{13} \mathrm{C}$ NMR: $\delta_{\mathrm{C}} 157.5$ (C-2), 133.5 (C-3), 177.4 (C-4), 161.1 (C-5), 99.6 (C-6), 165.4 (C-7), 94.6 (C-8), 156.9 (C-9), 104.5 (C-10), $121.5\left(\mathrm{C}-1^{\prime}\right), 131.2$ (C-2', C-6'), 115.3 (C-3', C-5'), 161.5 (C4'), 101.6 (C-1"), 74.4 (C-2"), 76.4 (C-3"), 69.8 (C-4"), 74.4 (C-5"), 63.0 (C-6"), 119.5 (C-1"'), 108.2 (C-2"', C-6"'), 145.6 (C-3"', C-5"'), 138.8 (C-4"'), 167.3 (C-7"'). Comparing the results with reference data [19], compound 17 was identified as kaempferol 3-O-(6-O-galloyl)- $\beta$-Dglucopyranoside.

Compound 18: ESI-MS: $m / z 471.2[\mathrm{M}+\mathrm{Na}]^{+} ;{ }^{1} \mathrm{H}$ NMR: $\delta_{\mathrm{H}}$ 6.23 (d, $J=2.0 \mathrm{~Hz}, \mathrm{H}-6), 6.42$ (d, $J=2.0 \mathrm{~Hz}, \mathrm{H}-8), 8.05$ (d, $\left.J=8.9 \mathrm{~Hz}, \mathrm{H}-2^{\prime}, \mathrm{H}-6^{\prime}\right), 6.93$ (d, J=8.9 Hz, H-3', H-5'), 5.28 (d, $\left.J=7.4 \mathrm{~Hz}, \mathrm{H}-1^{\prime \prime}\right), 3.49$ (t, $\left.J=8.5 \mathrm{~Hz}, \mathrm{H}-2^{\prime \prime}\right), 3.48$ (t, $J=8.5 \mathrm{~Hz}$, H-3"), 3.36 (t, $\left.J=9 \mathrm{~Hz}, \mathrm{H}-4^{\prime \prime}\right), 3.25$ (m, H-5"), 3.56 (dd, $\left.J=12.0,5.3 \mathrm{~Hz}, \mathrm{H}-6^{\prime \prime} \mathrm{a}\right), 3.70$ (dd, $\left.J=12.0,2.0 \mathrm{~Hz}, \mathrm{H}-6^{\prime \prime} \mathrm{b}\right){ }^{13} \mathrm{C}$ NMR: $\delta_{\mathrm{C}} 157.9$ (C-2), 134.0 (C-3), 178.0 (C-4), 161.0 (C-5), 98.7 (C-6), 164.5 (C-7), 93.7 (C-8), 157.0 (C-9), 104.3 (C10), 121.4 (C-1'), 130.9 (C-2',C-6'), 114.8 (C-3',C-5'), 160.0 (C-4'), $102.7\left(\mathrm{C}-1^{\prime \prime}\right), 74.2\left(\mathrm{C}-2^{\prime \prime}\right), 76.4\left(\mathrm{C}-3^{\prime \prime}\right), 69.8\left(\mathrm{C}-4^{\prime \prime}\right)$, $76.8\left(\mathrm{C}-5^{\prime \prime}\right), 61.1\left(\mathrm{C}-6^{\prime \prime}\right)$. Comparing the results with reference data [19], compound $\mathbf{1 8}$ was identified as astragalin.

Compound 19: Positive ESI-MS: $\mathrm{m} / \mathrm{z}$ 1115.5 $[\mathrm{M}+\mathrm{Na}]^{+} ;{ }^{1} \mathrm{H}$ NMR: $\delta_{\mathrm{H}} 6.27$ (d, $\left.J=8.3 \mathrm{~Hz}, \mathrm{H}-1\right), 5.63$ (t, $\left.J=9.2 \mathrm{~Hz}, \mathrm{H}-2\right)$, 5.94 (t, $J=9.3 \mathrm{~Hz}, \mathrm{H}-3$ ), 5.62 (t, $J=8.5 \mathrm{~Hz}, \mathrm{H}-4), 4.45$ (m, H5), 4.46 (m, H-6a), 4.56 (dd, $J=12.2,4.4 \mathrm{~Hz}, \mathrm{H}-6 \mathrm{~b}), 7.25$,

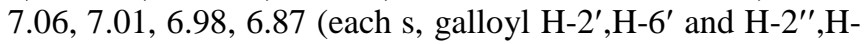
$6^{\prime \prime}$ ), 7.48, 7.32 (each d, $J=2.0 \mathrm{~Hz}, \mathrm{H}-2^{\prime}, \mathrm{H}^{\prime} 6^{\prime}$ of galloyl at C6); ${ }^{13} \mathrm{C}$ NMR: $\delta_{\mathrm{C}} 93.8(\mathrm{C}-1), 72.2(\mathrm{C}-2), 74.0(\mathrm{C}-3), 70.0(\mathrm{C}-$ 4), 74.3 (C-5), 63.5 (C-6), 121.1-119.7 (6xgalloyl C-1), 111.0-110.1 (6xgalloyl C-2, 6xgalloyl C-6), 144.9-147.9 (12xgalloyl C-3, C-5), 140.1-140.7 (6xgalloyl C-4), 166.2167.3 (6xgalloyl C-7). Comparing the results with Ref. [39], compound 19 was identified as 6-O-m-digalloyl-1,2,3,4tetra- $O$-galloyl- $\beta$-D-glucopyranoside.

Compound 25: Positive ESI-MS: $m / z$ 359.2 $[\mathrm{M}+\mathrm{Na}]^{+} ;{ }^{1} \mathrm{H}$ NMR: $\delta_{\mathrm{H}}(m$-isomer) 7.39 (d, $J=1.7 \mathrm{~Hz}, \mathrm{H}-2), 7.25$ (d, $J=1.7$ Hz, H-6), 3.48 (s, H-8), 7.23 (d, J=2.1 Hz, H-2'), 7.10 (d, $\left.J=2.1 \mathrm{~Hz}, \mathrm{H}-6^{\prime}\right)$, , (p-isomer) 7.20 (s, H-2, H-6), 3.86 (s, H8), 7.21 (s, H-2', H-6'); ${ }^{13} \mathrm{C}$ NMR: $\delta_{\mathrm{C}}(m$-isomer) 120.6 (C1), 117.3 (C-2), 147.6 (C-3), 139.7 (C-4), 146.6 (C-5), 114.7 (C-6), 166.3 (C-7), 52.5 (C-8), 121.4 (C-1'), 110.9 (C-2', C$\left.6^{\prime}\right), 146.4$ (C-3', C-5'), 140.0 (C-4'), 168.2 (C-7'), (p-isomer) 128.4 (C-1), 109.9 (C-2, C-6), 151.7 (C-3, C-5), 132.5 (C-4), $166.2(\mathrm{C}-7), 52.7(\mathrm{C}-8), 120.5\left(\mathrm{C}-1^{\prime}\right), 110.2\left(\mathrm{C}-2^{\prime}, \mathrm{C}-6^{\prime}\right)$, 146.3 (C-3',C-5'), 140.1 (C-4'), $168.2\left(\mathrm{C}^{\prime} 7^{\prime}\right)$. Comparing the results with Ref. [39], compound $\mathbf{2 5}$ was identified as an equilibrium mixture of methyl $m$-digallate and methyl $p$ digallate.
Compound 26: Positive ESI-MS: $m / z 373.3[\mathrm{M}+\mathrm{Na}]^{+} ;{ }^{1} \mathrm{H}$ NMR: $\delta_{\mathrm{H}}(m$-isomer) $7.41(\mathrm{~d}, J=2.1 \mathrm{~Hz}, \mathrm{H}-2), 7.28(\mathrm{~d}, J=2.1$ $\mathrm{Hz}, \mathrm{H}-6), 4.35$ (q, J=7.1 Hz, H-8), 1.39 (t, $J=7.1 \mathrm{~Hz}, \mathrm{H}-9$ ), 7.13 (s, H-2',H-6'), (p-isomer) 7.26 (d, J=2.1 Hz, H-2), 7.41 (d, $J=2.1 \mathrm{~Hz}, \mathrm{H}-6), 4.38$ (q, $J=7.1 \mathrm{~Hz}, \mathrm{H}-8), 1.38$ (t, $J=7.1$ $\mathrm{Hz}, \mathrm{H}-9), 7.24$ (s, H-2',H-6'); ${ }^{13} \mathrm{C}$ NMR: $\delta_{\mathrm{C}}(m$-isomer) 120.4 (C-1), 117.2 (C-2), 147.6 (C-3), 139.6 (C-4), 146.6 (C-5), 114.5 (C-6), 166.3 (C-7), 60.5 (C-8), 13.2 (C-9), 121.5 (C$\left.1^{\prime}\right), 109.3$ (C-2',C-6'), 146.4 (C-3',C-5'), 140.0 (C-4'), 168.2 (C-7'), (p-isomer) 128.4 (C-1), 109.9 (C-2,C-6), 151.7 (C3,C-5), 132.5 (C-4), 166.2 (C-7), 52.7 (C-8), 13.3 (C-9), $120.5\left(\mathrm{C}-1^{\prime}\right), 110.2$ (C-2',C-6'), 146.3 (C-3',C-5'), 140.1 (C$\left.4^{\prime}\right), 168.2\left(\mathrm{C}-7^{\prime}\right)$. Comparing the results with Ref. [40], compound 26 was identified as equilibrium mixture of ethyl $m$-digallate and ethyl $p$-digallate.

The major components purified from the EtOAc part were compounds $16(1.94 \%), 7(1.30 \%)$, and $4(0.58 \%)$, whereas in the $\mathrm{H}_{2} \mathrm{O}$ part, the major components were compounds $\mathbf{2 4}$ $(0.33 \%)$, and $4(0.29 \%)$.

Compounds $1,7,8,9,16,17,18,20,21$, and 22 were previously identified in the methanol extract of $P$. lactiflora flowers and compounds $1,4,8,9,14,16,18$ and 21 in the methanol extract of $P$. suffruticosa flowers and in these cases, compounds 7, 8 and 16 were the major compounds [18]. Compounds 1, 4, 8, 9, 11, 15, 16 and 19 were previously identified in the roots of $P$. lactiflora $[15,26,35]$. To our knowledge, the other compounds were identified for the first time in P. lactiflora flowers.

\subsection{HPLC Analysis of the 70\% EtOH Extract}

On the ultraviolet spectra of HPLC-UV chromatograms of compounds detected in $P$. lactiflora, maximum absorbance values were observed around 205, 232, 278, and $340 \mathrm{~nm}$. Hence characteristic chromatographic patterns were obtained by using $205 \mathrm{~nm}$ as the detection wavelength. There were 16 characteristic peaks found in the chromatogram, which covered more than $90 \%$ of the total area (Fig. 2). Sixteen components were identified by comparing their retention time and UV spectrum with those of isolated pure compounds $(2-5,7,8,11,13-19,25$, and 26) which were eluted in parallel under the same conditions. Major components of $70 \% \mathrm{EtOH}$ extract (peack area at $205 \mathrm{~nm}$ ) were composed of 16, 17, 18, 19, 25, and 26 (Fig. 2).

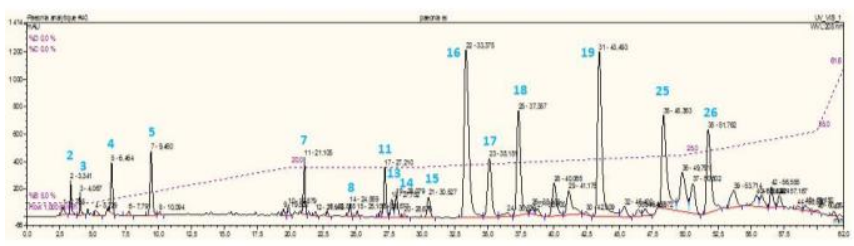

Figure 2: HPLC chromatographic profile of the $70 \% \mathrm{EtOH}$ extract of P. lactiflora flowers.

\subsection{DPPH Free Radical Scavenging Activity}

The $70 \%$ EtOH from $P$. lactiflora exhibited significant scavenging effect on DPPH radicals $\left(\mathrm{IC}_{50} 2.3 \mu \mathrm{g} \cdot \mathrm{mL}^{-1}\right)$ compared to ascorbic acid used as positive control ( $\mathrm{IC}_{50} 11.3$ $\mu \mathrm{g} \cdot \mathrm{mL}^{-1}$, respectively) (Table 1 ). To identify the compounds responsible for the observed activity, this extract was dissolved in $\mathrm{H}_{2} \mathrm{O}$ and partitioned with EtOAc. The organic 
phase was about 10 times more active than the crude extract $\left(\mathrm{IC}_{50}=0.3 \mu \mathrm{g} \cdot \mathrm{mL}^{-1}\right)$.

Compounds 7, 15, 16, 25, and 26 isolated from the EtOAc phase exhibited good scavenging effect on DPPH radicals with $\mathrm{IC}_{50}$ ranging from 0.48 to $12.8 \mu \mathrm{g} \cdot \mathrm{mL}^{-1}$ (Table 1 ). Compound 7 was the most potent $\left(\mathrm{IC}_{50} 0.48 \mu \cdot \mathrm{mL}^{-1}\right)$ However, the fact that the $\mathrm{IC}_{50}$ values of the nine phenolic compounds isolated from EtOAc extract were higher than that of the crude EtOAc extract $\left(0.3 \mu \mathrm{g} \cdot \mathrm{mL}^{-1}\right)$, could be related to the combination effects or to more potent components in the crude EtOAc extract yet to be discovered as lead natural antioxidants by further chemical research. On the other hand, the hydrophilic phase had a relatively lower scavenging effect than the $70 \% \mathrm{EtOH}$ extract $\left(\mathrm{IC}_{50}=86.7\right.$ $\left.\mu \mathrm{g} \cdot \mathrm{mL}^{-1}\right)$ and the most active compounds isolated from this phase were $\mathbf{1 1}, \mathbf{1 5}, \mathbf{2 0}$, and $\mathbf{2 1}$ with $\mathrm{IC}_{50}$ ranging from 2.37 to $5.2 \mu \mathrm{g} \cdot \mathrm{mL}^{-1}$ (Table 1$)$.

Table 1. $\mathrm{IC}_{50}$ values of mushroom tyrosinase inhibition and antioxidant effects of $P$. lactiflora flowers extracts and compounds. Data were expressed as a mean value of three independent experiments.

\begin{tabular}{|c|c|c|}
\hline & $\begin{array}{l}\mathrm{DPPH}^{+} \text {scavenging } \\
\mathrm{IC}_{50}\left(\mu \mathrm{g} \cdot \mathrm{mL}^{-1}\right)\end{array}$ & $\begin{array}{l}\text { Tyrosinase } \\
\mathrm{IC}_{50}\left(\mu \mathrm{g} \cdot \mathrm{mL}^{-1}\right)\end{array}$ \\
\hline 1 & 1.80 & $>200$ \\
\hline 2 & $>200$ & $>200$ \\
\hline 3 & 7.07 & 48 \\
\hline 4 & 4.23 & 45 \\
\hline 5 & 4.07 & 47 \\
\hline 6 & $>200$ & $>200$ \\
\hline 7 & 0.48 & $>200$ \\
\hline 8 & $>200$ & $>200$ \\
\hline 9 & $>200$ & $>200$ \\
\hline 10 & $>200$ & $>200$ \\
\hline 11 & 2.37 & 41.0 \\
\hline 12 & $>200$ & $>200$ \\
\hline 13 & $>200$ & $>200$ \\
\hline 14 & $>200$ & $>200$ \\
\hline 15 & 3.10 & 0.23 \\
\hline 16 & 4.52 & 0.38 \\
\hline 17 & 7.83 & 0.35 \\
\hline 18 & $>200$ & $>200$ \\
\hline 19 & 12.0 & 37 \\
\hline 20 & 4.53 & $>200$ \\
\hline 21 & 5.20 & 46 \\
\hline 22 & $>200$ & $>200$ \\
\hline 23 & $>200$ & $>200$ \\
\hline 24 & 12.8 & 34 \\
\hline 25 & 1.63 & $>200$ \\
\hline 26 & 2.03 & $>200$ \\
\hline EtOH extract & 2.3 & 0.35 \\
\hline EtOAc extract & 0.3 & 0.35 \\
\hline $\mathrm{H}_{2} \mathrm{O}$ extract & 86.7 & 0.35 \\
\hline Kojic acid ${ }^{b}$ & - & 6.4 \\
\hline Ascorbic acid ${ }^{b}$ & 11.3 & - \\
\hline
\end{tabular}

${ }^{\mathrm{a}}$ NA: $50 \%$ inhibition not achieved at the concentration of 200 $\mu \mathrm{g} \cdot \mathrm{mL}^{-1}$

${ }^{\mathrm{b}}$ Used as a positive control.

\subsection{Fungal Tyrosinase Inhibition}

The whitening activity of $70 \% \mathrm{EtOH}, \mathrm{H}_{2} \mathrm{O}$ and EtOAc extracts was evaluated, in addition to compounds 1-26 by using the fungal tyrosinase inhibitory assay. We found that $70 \% \mathrm{EtOH}, \mathrm{H}_{2} \mathrm{O}$ and EtOAc extracts possess good tyrosinase inhibitory activity with $\mathrm{IC}_{50} 0.35 \mu \mathrm{g} \cdot \mathrm{mL}^{-1}$ for each one compared to kojic acid used as positive control $\left(\mathrm{IC}_{50} 6.4\right.$ $\left.\mu \mathrm{g} \cdot \mathrm{mL}^{-1}\right)$. Compounds $\mathbf{1 5 - 1 7}$ isolated from the EtOAc extract reduce significantly fungal tyrosinase activity with $\mathrm{IC}_{50}$ ranging from 0.23 to $0.35 \mu \mathrm{g} \cdot \mathrm{mL}^{-1}$ (Table 1 ). Only compound $\mathbf{2 4}$ isolated from the hydrophilic phase exhibited moderate tyrosinase inhibitory activity $\left(\mathrm{IC}_{50} 34 \mu \mathrm{g} \cdot \mathrm{mL}^{-1}\right)$. Compound $\mathbf{1 5}$ was the most active $\left(\mathrm{IC}_{50} 0.23 \mu \mathrm{g} \cdot \mathrm{mL}^{-1}\right)$ compared to kojic acid $\left(\mathrm{IC}_{50} 6.4 \mu \mathrm{g} \cdot \mathrm{mL}^{-1}\right)$ used as positive control (Table 1).

\section{CONCLUSION}

In summary, 26 known compounds were isolated from the flowers of Paeonia lactiflora. A simple, accurate, and reliable method was developed to evaluate the quality of $P$. lactiflora extracts by using the established HPLC fingerprint and the determination of sixteen compounds. This method might find its applicability to the determination of regional variation of the active compounds and to a cultivation management for $P$. lactiflora. The antioxidant assays revealed that $70 \% \mathrm{EtOH}$ extract and EtOAc phase had significant antioxidant abilities in DPPH scavenging activity. The most active compound was identified as methyl gallate (7). Through the in vitro fungal tyrosinase inhibition screening, we found, that $70 \% \mathrm{EtOH}$, EtOAc, and $\mathrm{H}_{2} \mathrm{O}$ extracts and compounds 15, 16 and 17 inhibit the target protein activities with good $\mathrm{IC}_{50}$ values. It is interesting that the tetra- and penta-galloylglucoses (15 and 16) and the flavonoid 17 have bi-functionality, not just have antioxidative abilities, but also had the inhibitory effects on fungal tyrosinase. These constituents from $P$. lactiflora exhibited potential applications in medical cosmetology and food supplementation, simultaneously.

\section{CONFLICT OF INTEREST}

The authors confirm that this article content has no conflict of interest.

\section{ACKNOWLEDGEMENTS}

The authors are grateful to CNRS, Conseil Régional Champagne Ardenne, Conseil Général de la Marne, and Ministry of Higher Education and Research (MESR), the PIANET CPER project, and to LVMH recherche for financial support.

\section{REFERENCES}

[1] Alliwell, B.; Gutteridge, J.M.C. Oxygen toxicity, oxygen radicals, transition metals and disease. Biochem. J., 1984, 219, 1-4.

[2] Fantone, J.C.; Ward, P.A. Role of oxygen-derived free radicals and metabolites in leukocyte-dependent inflammatory reactions. Am. J. Pathol., 1982, 107, 395-418.

[3] Yehye, A.W.; Abdul Rahman, N.; Ariffin, A.; Abd Hamid, S.; Alhadi, A.; Kadir, F.; Yaeghoobi, M. Understanding the chemistry behind the antioxidant activities of butylated hydroxytoluene (BHT). Eur J Med Chem., 2015, 101, 295-312.

[4] Sies, H. Biochemistry of oxidative stress. Angew. Chem. Int. Edit., 2003, 25, 1058-1071. 
[5] Velioglu, Y.S.; Mazza, G.; Gao, L.; Oomah, B.D. Antioxidant activity and total phenolics in selected fruits, vegetables, and grain products. J. Agri. Food. Chem., 1998, 46, 4113-4117.

[6] Hilton, J. Antioxidants: function, types and necessity of inclusion in pet foods. Can. Vet. J., 1989, 30, 682-684.

[7] Hirose, M.; Takesada, Y.; Tanaka, H.; Tamano, S.; Kato, T.; Shirai, T. Carcinogenicity of antioxidants BHA, caffeic acid, sesamol, 4methoxyphenol and catechol at low doses, either alone or in combination and modulation of their effects in a rat medium-term multi-organ carcinogensis model. Carcinogenesis, 1998, 19, 207-212.

[8] Briganti, S.; Camera, E.; Picardo, M. Chemical and instrumental approaches to treat hyperpigmentation. Pigment. Cell. Res., 2003, 16, 101-110.

[9] Parvez, S.: Kang, M.; Chung, H.S.; Bae, H. Naturally occurring tyrosinase inhibitors: mechanism and applications in skin health, cosmetics and agriculture industries. Phytother. Res., 2007, 21, 805816.

[10] K. Maeda, M. Fukuda, 'In vivo effectiveness of several whitening cosmetic components in human melanocytes', J. Soc. Cosmet. Chem. 1991, 42, 361-368.

[11] Yoshikawa, M.; Uchida, E.; Kawaguchi, A.; Kitagawa, I.; Yamahara, J. Galloyloxypaeoniflorin, suffruticoside-A, suffruticoside-B, suffruticoside-C, and suffruticoside-D, 5 new antioxidative glycosides, and suffruticoside-E, a paeonol glycoside, from Chinese Moutan cortex. Chem. Pharm. Bull., 1992, 40, 2248-50.

[12] Matsuda, H.; Ohta, T.; Kawaguchi, A.; Yoshikawa, M. Structures and radical scavenging effects of suffruticosides $\mathrm{A}, \mathrm{B}, \mathrm{C}, \mathrm{D}$, and $\mathrm{E}$ and galloyloxypaeoniflorin. Chem. Pharm. Bull., 2001, 49, 69-72.

[13] Tanaka, T.; Fukumori, M.; Ochi, T.; Kouno, I. Paeonianins A-E, new dimeric and monomeric ellagitannins from the fruits of Paeonia lactiflora. J. Nat. Prod., 2003, 66, 759-763.

[14] Qiu, J.; Chen, M.; Liu, J.; Huang, X.; Chen, J.; Zhou, L.; Ma, J.; Sexitus, P.; Pena, A.M.; Cai, Z.; Jeulin, S. The skin-depigmenting potential of Paeonia lactiflora root extract and paeoniflorin: in vitro evaluation using reconstructed pigmented human epidermis. Int. J. Cosmet. Sci., 2016, 38,444-451.

[15] Parker, S.; May, B.; Zhang, C.; Zhang, L.A.; Lu, C.; Xue, C.A. Pharmacological Review of Bioactive Constituents of Paeonia lactiflora Pallas and Paeonia veitchii Lynch. Phytother. Res., 2016, $30,1445-1473$.

[16] He, C.N.; Peng, Y.; Zhang, Y.C.; Xu, L.J.; Gu, J.; Xiao, P.G.; Phytochemical and biological studies of Paeoniaceae. Chem. Biodivers., 2010, 7, 805-38.

[17] Murakami, N.; SaKa, M.; Shimada, H.; Matsuda, H.; Yamahara, J.; Yoshikawa, M. New bioactivemonoterpene glycosides from Paeoniae radix. Chem. Pharm. Bull., 1996, 44, 1279-1281.

[18] Liu, X.; Yang, M.H.; Wang, X.B.; Xie, S.S.; Li, Z.R.; Kim, D.H.; Park, J.S.; Kong, L.Y. Lignans from the root of Paeonia lactiflora and their anti- $\beta$-amyloid aggregation activities. Fitoterapia, 2015, $103,136-142$.

[19] Shu, X.; Duan, W.; Liu, F.; Shi, X.; Geng, Y.; Wang, X.; Yang, B. Preparative separation of polyphenols from the flowers of Paeonia lactiflora Pall. by high-speed counter-current chromatography. $J$. Chromatogr. B, 2014, 947, 62-67.

[20] Ogawa, K.; Nakamura, S.; Sugimoto, S.; Tsukioka, J.; Hinomaru, F.; Nakashima, T. Matsumoto, T. Ohta, K. Fujimoto, M. Yoshikawa, H. Matsuda, S. Constituents of flowers of Paeoniaceae plants, Paeonia suffruticosa and Paeonia lactiflora. Phytochem. Lett., 2015, 12, 98104.

[21] Jin, Y.S.; Chen, J.C.; Jin, Y.; Li, C.; Chen, M.; Tao, J. Antioxidant and anti-inflammatory activities of ethyl ether extract from Paeonia lactiflora Pall. flowers. Asian J. Chem., 2016, 28, 1144-1148.

[22] He, X., Li, J., Zhao, W., Liu, R., Zhang, L., Kong, X. Chemical fingerprint analysis for quality control and identification of Ziyang green tea by HPLC. Food Chem., 2015, 171, 405-411.
[23] Bendaikha, S.; Gadaut, M.; Harakat, D.; Alabdul Magid, A. Acylated flavonol glycosides from the flower of Elaeagnus angustifolia L. Phytochemistry, 2014, 103, 129-13. [23] Gossan, D.P.A., Alabdul Magid, A., Yao-Kouassi, P.A., Josse, J., Gangloff, S.C., Morjani, H., Voutquenne-Nazabadioko, L. Antibacterial and cytotoxic triterpenoids from the roots of Combretum racemosum. Fitoterapia, 2016, 110, 89-95.

[24] Markham, K.; Terna, B.; Stanely, R.; Geiger, H.; Mabry, T. Carbon13 NMR studies of flavonoids-II. Tetrahedron, 1978, 34, 1389-1397.

[25] Kashiwada, Y.; Nonaka, G.I.; Noshioka, I. Galloylsucroses from rhubarbs. Phytochemistry, 1988, 27, 1469-1472.

[26] Subeki, H.M.; Kosaku, T.; Masahiro, Y.; Osamu, Y.; Yoshimitsu, M.; Ken, K.; Sumiko, K.; Trimurningsih, C.; teruhiko, Y. Anti-babesial and anti-plasmodial compounds from Phyllanthus niruri. J. Nat. Prod., 2005, 68, 537-539.

[27] Hsu F.L.; Lee, Y.Y.; Cheng, J.T. Antihypertensive activity of 6-Ogalloyl-D-glucose, a phenolic glycoside from Sapium sebiferum. Phytochemistry, 1994, 57, 308-312.

[28] Fkyerat, A.; Burki, N.; Tabacchi, R. Enantioselective synthesis of 3 hydroxycitronellic acid isolated from Ceratocystis fimbriata sp. Platani. Tetrahedron: Asymmetry, 1996, 7, 2023-2028.

[29] Lin, H.C.; Ding, H.Y.; Wu, T.S.; Wu, P.L. Monoterpene glycosides from Paeonia suffruticosa. Phytochemistry, 1996, 41, 237-242.

[30] Kaneda, M.; Itaka, Y.; Shibata, S. Absiolute structure of paeoniflorin, albiflorin, oxypaeoniflorin, and benzoylpaeoniflorin isolated from Chinese paeony root. Tetrahedron, 1972, 28, 4309-4317.

[31] Hase, T.; Kawamoto, Y.; Ohtani, K.; Kasai, R.; Yamasaki, K.; Picheansoonthon, C. Cyclohexylethanoids and related glucosides from Millingtonia hortensis. Phytochemistry, 1995, 39, 235-241.

[32] Ma, S.J.; Mizutani, M.; Hiratake, J.; Hayashi, K.; Yagi, K.; Watanabe, N.; Sakata, K. Substrate specificity of betaprimeverosidase, a key enzyme in aroma formation during oolong tea and black tea manufacturing. Biosci. Biotechnol. Biochem., 2001, 65, 2719-2729.

[33] Bonacheva, V.M.; Botirov, E.K. Kaempferol and its glycosides from Equisetum silvaticum L. from the Khanty Mansi autonomous area. Russ. J Bioorg. Chem., 2014, 40, 777-780.

[34] Diaz, J.G.; Carmona, A.J.; Torres, F.; Quintana, J.; Estévez, F.; Herz, W. Cytotoxic activities of flavonoid glycoside acetates from Consolida oliveriana. Planta Med., 2008, 74, 171-174.

[35] Ozden, S.; Durust, N.; Toki, K.; Saito, N.; Honda, N. Acylated kaempferol glycosides from the flowers of Delphinium formosum Phytochemistry, 1998, 49, 241-245.

[36] Zhang, X.Q.; Gu, H.M.; Li, X.Z.; Xu, Z.N.; Chen, Y.S.; Li, Y. AntiHelicobacter pylori compounds from the ethanol extracts of Geranium wilfordii. J. Ethnopharmacol., 2013, 147, 204-207.

[37] Bing, S.J.; Kim, M.J.; Park, E.; Ahn, G.; Kim, D.S.; Ko, R.K.; Lee, N.H.; Shin, T.; Park, J.W.; Jee, Y. 1,2,3,4,6-penta- $O$-galloyl- $\beta$-Dglucose protects splenocytes against radiation-induced apoptosis in murine splenocytes' Biol. Pharm. Bull. 2010, 33, 1122-1127.

[38] Nishizawa, M.; Yamagishi, T.; Nonaka, G.; Nishioka, I.; Structure of gallotannins in Paeoniae radix. Chem. Pharm. Bull. 1980, 28, 28502852.

[39] Horgen, F.D.; Madulid, D.A.; Angerhofer, C.K.; Pezzuto, M.; Soejarto, D.D.; Farnsworth, N.R. Isolation of gallic acid esters as antiplasmodial constituents of Swintonia foxworthyi (Anacardiaceae). Phytomedicine, 1997, 4, 353-356.

[40] Dorta, E.; Gonzales, M.; Lob, M.G.; Sanchez-Moreno, C.; De Ancos, B. Screening of phenolic compounds in by-product extracts from mangoes (Mangifera indica L.) by HPLC-ESI-QTOF-MS and multivariate analysis for use as a food ingredient. Food Res. Int., 2014, $57,51-60$ 